\title{
p113 isoform encoded by CUX1 circular RNA drives tumor progression via facilitating ZRF1/ BRD4 transactivation
}

\author{
Feng Yang ${ }^{1 \dagger}$, Anpei Hu ${ }^{1 \dagger}$, Yanhua Guo ${ }^{1 \dagger}$, Jianqun Wang ${ }^{1 \dagger}$, Dan $\mathrm{Li}^{1}$, Xiaojing Wang ${ }^{2,3}$, Shikai Jin ${ }^{1}$, Boling Yuan',
} Shuang Cai ${ }^{4}$, Yi Zhou ${ }^{4}$, Qilan $\mathrm{Li}^{1}$, Guo Chen ${ }^{1}$, Haiyang Gao ${ }^{5}$, Liduan Zheng ${ }^{2,4^{*}}$ and Qiangsong Tong ${ }^{1,2^{*}}$ (D)

\begin{abstract}
Background: Metabolic reprogramming sustains tumorigenesis and aggressiveness of neuroblastoma (NB), the most common extracranial malignancy in childhood, while underlying mechanisms and therapeutic approaches still remain elusive.

Methods: Circular RNAs (circRNAs) were validated by Sanger sequencing. Co-immunoprecipitation, mass spectrometry, chromatin immunoprecipitation (ChIP) sequencing, and RNA sequencing assays were applied to explore protein interaction and target genes. Gene expression regulation was observed by ChIP, dual-luciferase reporter, real-time quantitative RT-PCR, and western blot assays. Gain- and loss-of-function studies were performed to observe the impacts of circRNA-encoded protein and its partners on the lipid metabolism, mitochondrial activity, growth, invasion, and metastasis of NB cells.

Results: A novel 113-amino acid protein (p113) of CUT-like homeobox 1 (CUX1) was identified in NB cells treated by serum deprivation. Further validating studies revealed that nuclear p113 was encoded by circRNA of CUX1, and promoted the lipid metabolic reprogramming, mitochondrial activity, proliferation, invasion, and metastasis of NB cells. Mechanistically, p113 interacted with Zuotin-related factor 1 (ZRF1) and bromodomain protein 4 (BRD4) to form a transcriptional regulatory complex, and mediated the transactivation of ZRF1/BRD4 in upregulating ALDH3A1, NDUFA7, and NDUFAF5 essential for conversion of fatty aldehydes into fatty acids, fatty acid $\beta$-oxidation, and mitochondrial complex I activity. Administration of an inhibitory peptide blocking p113-ZRF1 interaction suppressed the tumorigenesis and aggressiveness of NB cells. In clinical NB cases, high expression of p113, ZRF1, or BRD4 was associated with poor survival of patients.
\end{abstract}

Conclusions: These results indicate that p113 isoform encoded by CUX1 circular RNA drives tumor progression via facilitating ZRF1/BRD4 transactivation.

\footnotetext{
*Correspondence: Id_zheng@hotmail.com; qs_tong@hotmail.com ${ }^{\dagger}$ Feng Yang, Anpei Hu, Yanhua Guo and Jianqun Wang contributed equally to this work.

${ }^{2}$ Clinical Center of Human Genomic Research, Union Hospital, Tongji Medical College, Huazhong University of Science and Technology, 1277 Jiefang Avenue, Wuhan, Hubei Province 430022, People's Republic of China

${ }^{3}$ Department of Geriatrics, Union Hospital, Tongji Medical College Huazhong University of Science and Technology, 1277 Jiefang Avenue, Wuhan, Hubei Province 430022, People's Republic of China

Full list of author information is available at the end of the article
} permits use, sharing, adaptation, distribution and reproduction in any medium or format, as long as you give appropriate credit to the original author(s) and the source, provide a link to the Creative Commons licence, and indicate if changes were made. The images or other third party material in this article are included in the article's Creative Commons licence, unless indicated otherwise in a credit line to the material. If material is not included in the article's Creative Commons licence and your intended use is not permitted by statutory regulation or exceeds the permitted use, you will need to obtain permission directly from the copyright holder. To view a copy of this licence, visit http://creativecommons.org/licenses/by/4.0/. The Creative Commons Public Domain Dedication waiver (http://creativeco mmons.org/publicdomain/zero/1.0/) applies to the data made available in this article, unless otherwise stated in a credit line to the data. 
Keywords: Circular RNA-coding protein, Zuotin-related factor 1, Bromodomain protein 4, Neuroblastoma

progression

\section{Background}

Neuroblastoma (NB), a solid malignancy featured by rapid progression and high mortality, accounts for more than $15 \%$ of tumor-related deaths in pediatric population [1]. For high-risk NB patients, the clinical outcome still remains unfavorable in despite of multimodal therapeutics [1]. Beside aerobic glycolysis and glutaminolysis, tumor cells obtain energies from lipid metabolism for thriving in challenging environments, including uptake of fatty acids, de novo lipid synthesis [2], and fatty acid $\beta$-oxidation (FAO) [3]. FAO allows for mitochondrial conversion of long-chain fatty acids into acetyl-CoA, which is subsequently oxidized via tricarboxylic acid cycle and electron transport chain (ETC) to produce ATP [4]. FAO pathway is dysregulated in diverse human malignancies [3], and tumor cells rely on FAO for proliferation, survival, stemness, drug resistance, or metastasis [5]. However, transcriptional regulators of lipid metabolic reprogramming in NB still remain largely elusive.

Circular RNAs (circRNAs) are a subclass of non-coding RNAs (ncRNAs) with closed continuous loops, and act as key regulators of gene expression in cancers by serving as miRNA (microRNA) sponges or RNA-binding protein partners [6]. For example, CDR1as exerts miRNA sponging activity as a competing endogenous RNA [7]. Eukaryotic translation elongation factor $3 \mathrm{~J}$ (EIF3J)derived circRNA (circ-EIF3) interacts with U1 snRNP and RNA polymerase II to facilitate parental gene transcription [8]. Circ-AMOTL1 derived from angiomotinlike 1 (AMOTL1) promotes tumorigenesis by increasing nuclear retention of c-Myc [9]. Meanwhile, some circRNAs have the capability to encode peptides or proteins [10-13]. For example, circRNA-coding protein FBXW7185aa inhibits glioblastoma proliferation by reducing c-Myc stabilization [10]. A novel protein encoded by circular RNA of AKT serine/threonine kinase 3 ( $\operatorname{circAKT3)}$ suppresses glioblastoma tumorigenesis by competing with active phosphoinositide-dependent kinase 1 [12]. Meanwhile, $\beta$-catenin circular RNA (circ $\beta$-catenin)derived protein promotes growth of liver cancer cells via activating Wnt pathway [11]. Circular RNA of protein phosphatase 1 regulatory subunit 12A ( $\operatorname{circPPP1R12A})$ encodes a protein that drives metastasis of colon cancer via Hippo-YAP signaling pathway [13]. However, the roles and underlying mechanisms of circRNA-coding proteins in NB remain to be determined.

In this study, we identify a 113-amino acid protein (p113) encoded by circRNA of CUT-like homeobox 1
(CUX1) as a driver of NB progression. Mechanistically, p113 interacts with Zuotin-related factor 1 (ZRF1) and bromodomain protein 4 (BRD4) to form a transcriptional regulatory complex, resulting in upregulation of aldehyde dehydrogenase 3 family member A1 (ALDH3A1), NADH:ubiquinone oxidoreductase subunit A1 (NDUFA1), and NADH: ubiquinone oxidoreductase complex assembly factor 5 (NDUFAF5) essential for conversion of fatty aldehydes into fatty acids, FAO, and mitochondrial complex I activity for ATP production. Administration of an inhibitory peptide blocking p113ZRF1 interaction suppresses FAO, mitochondrial complex I activity, tumorigenesis, and aggressiveness of NB cells, indicating the oncogenic roles of p113 and ZRF1 in lipid metabolic reprogramming and NB progression.

\section{Materials and methods}

\section{Cell culture}

Human NB cell lines SH-SY5Y (CRL-2266), SK-N-SH (HTB-11), SK-N-BE(2) (CRL-2271), BE(2)-C (CRL-2268), and IMR-32 (CCL-127), cervical cancer HeLa (CCL-2) cells, prostate cancer PC-3 cells (CRL-1435), nontransformed mammary epithelial MCF 10A (CRL-10317), and embryonic kidney HEK293T (CRL-3216) were obtained from American Type Culture Collection (Rockville, MD). Cell lines were authenticated by short tandem repeat profiling, and used within 6 months after resuscitation. Mycoplasma was regularly examined with Lookout Mycoplasma PCR Detection Kit (Sigma, St. Louis, MO). Tumor cells and HEK293T cells were cultured in RPMI1640 supplied with $10 \%$ fetal bovine serum (Gibco, Grand Island, NY), while MCF 10A cells were cultured in DMEM/F12 medium containing $5 \%$ horse serum (Invitrogen, Carlsbad, CA) and $20 \mathrm{ng} / \mathrm{ml}$ epidermal growth factor (Peprotech, Rocky Hill, NJ). For metabolic SD stress, cells were maintained in RPMI1640 containing 1\% fetal bovine serum.

\section{RT-PCR and real-time quantitative PCR}

Nuclear, cytoplasmic, and total RNAs were extracted using RNA Subcellular Isolation Kit (Active Motif, Carlsbad, CA) or RNeasy Mini Kit (Qiagen Inc., Redwood City, CA). For circRNA detection, RNase R (3U/ug, Epicenter, Madison, WI) treatment was undertaken at $37^{\circ} \mathrm{C}$ for $15 \mathrm{~min}$, while reverse transcription kit (TakaRa, Dalian, China) was used for cDNA synthesis. Quantification of mRNA and circRNA was performed using a SYBR Green PCR Master Mix (Applied Biosystems, Carlsbad, CA) and primers (Additional file 1: Table S1). 


\section{Western blot assay}

Proteins were extracted with RIPA lysis buffer (Thermo Fisher Scientific, Inc., Waltham, MA). Anti-p113 polyclonal antibody was prepared by immunizing rabbits with synthesized peptide corresponding to C-terminus of p113 (EQQLSAKNSTLKGRRD; ABclonal Biotechnology Co., Ltd., Wuhan, China). Western blot analysis was performed as previously described [14-16], with antibodies specific for CUX1 (ab230844), transcription factor 3 (TCF3, ab69999), ALDH3A1 (ab186726), NDUFA1 (ab249923), NDUFAF5 (ab240971), $\beta$-actin (ab179467, Abcam Inc., Cambridge, MA), CUX1 (sc-514,008), histone H3 (sc-517,576), glyceraldehyde 3-phosphate dehydrogenase (GAPDH, sc-47724), glutathione S-transferase (GST, sc-33614, Santa Cruz Biotechnology, Santa Cruz, CA), Flag-tag (14793S), ZRF1 (12844S), BRD4 (13440S), hemagglutinin (HA)-tag (3724S), or His-tag (12698S, Cell Signaling Technology Inc., Danvers, MA).

\section{Plasmid construction and stable transfection}

Linear ecircCUX1 (hsa_circ_30402) was obtained from NB tissues by PCR (Additional file 1: Table S2) and inserted into pLCDH-ciR (Geenseed Biotech Co., Guangzhou, China). The ecircCUX1-3Flag construct was established by inserting a $3 \times$ Flag-coding sequence upstream of TGA codon within linear ecircCUX1 (Additional file 1: Table S2). Mutation of ecircCUX1 was performed with GeneTailor $^{\mathrm{TM}}$ Site-Directed Mutagenesis System (Invitrogen) and primers (Additional file 1: Table S2). Synthesized p113-3Flag sequence was subcloned into pcDNA3.1mini (Addgene, Cambridge, MA), while p113-3Flag with circular frames was ligated into CV186 (Genechem Co., Ltd., Shanghai, China) or pCMV-HA (Addgene). Human ZRF1 cDNA (1866bp) was obtained from Genechem Co., Ltd., while BRD4 cDNA (4089bp) was provided by Dr. Guosong Jiang [17]. Truncations of ZRF1 or BRD4 were obtained by PCR amplification (Additional file 1: Table S2) and subcloned into pCMV-3Tag-1A (Stratagene, Santa Clara, CA), pCDNA4-His (Invitrogen), pGEX-6P-1 (Addgene), or pMal-c4X (Addgene), respectively. The pGEX-6P-1 or pMal-c4X constructs were transformed into $E$. coli to produce GST-tagged ZRF1 or maltose-binding protein (MBP)-tagged BRD4 proteins. Single guide RNAs (sgRNAs) targeting downstream region of gene transcription start site (Additional file 1: Table S3) were inserted into dCas9-BFP-KRAB (Addgene) [18]. Oligonucleotides specific for short hairpin RNAs (shRNAs, Additional file 1: Table S3) were inserted into GV298 (Genechem Co., Ltd). Lentiviral plasmids were co-transfected with psPAX2 and pMD2G into HEK293T cells to generate infectious lentivirus. Stable cell lines were obtained with puromycin selection for 3-4 weeks.

\section{Dual-luciferase reporter assay}

The internal ribosome entry site (IRES) reporter of ecircCUX1 was amplified with primers (Additional file 1: Table S2) and subcloned into pGL3-Basic (Promega, Madison, WI). Human ZRF1 activity reporter was established by inserting oligonucleotides containing four canonical binding sites (Additional file 1: Table S2) into pGL3-Basic (Promega). Promoter fragment of ALDH3A1 (1179bp), NDUFA1 (1077bp), or NDUFAF5 (1079bp) was amplified from genomic DNA with primers (Additional file 1: Table S2) and subcloned into pGL3-Basic (Promega). Dual-luciferase assay was performed according to the manufacturer's instructions (Promega).

\section{Knockin with CRISPR-Cas9 genome editing}

Genomic insertion of a $3 \times$ Flag coding sequence or mutation of ecircCUX1 was performed by homologydirected repair of a CRISPR-Cas9-induced DNA break. The sgRNA oligonucleotides targeting exon 9 of CUX1 were designed using online program (http://crispr.mit. edu), and ligated into LentiCRISPR V2 vector (Addgene). Donor DNA carrying $1200 \mathrm{bp}$ of homologous sequences upstream and downstream exon 9 of CUX1 with $3 \times$ Flag insertion or open reading frame (ORF) mutation was synthesized. Cells were co-transfected with sgRNA and donor DNA, selected with puromycin for 2-3 weeks, and validated by PCR amplification using primers (Additional file 1: Table S1) and Sanger sequencing.

\section{RNA sequencing (RNA-seq)}

Total RNA of tumor cells $\left(1 \times 10^{6}\right)$ was isolated using $\mathrm{TRIzol}^{\mathrm{TM}}$ reagent (Life Technologies, Inc., Gaithersburg, $\mathrm{MD})$. Library preparation and transcriptome sequencing on a BGIseq500 platform were carried out at Beijing Genomics Institute (BGI-Shenzhen, China).

\section{Co-immunoprecipitation and mass spectrometry}

Co-immunoprecipitation (co-IP) was performed as previously described [14-16], with antibodies for p113 (ABclonal Biotechnology Co., Ltd), GST-tag (sc-33614, Santa Cruz Biotechnology), Flag-tag (14793S), ZRF1 (12844S), BRD4 (13440S), HA-tag (3724S), His-tag (12698S), MBP-tag (2396S, Cell Signaling Technology Inc., MA). Bead-bound proteins were analyzed by Coomassie blue staining, western blot, or mass spectrometry (Wuhan Institute of Biotechnology, Wuhan, China).

\section{Chromatin immunoprecipitation (ChIP) and ChIP sequencing (ChIP-seq)}

ChIP assay was undertaken using EZ-ChIP kit (Upstate Biotechnology, Temacula, CA) [14-16], with antibodies specific for p113 (ABclonal Biotechnology Co., Ltd), ZRF1 (12844S), or BRD4 (13,440, Cell Signaling Technology 
Inc.) and primers (Additional file 1: Table S1). ChIP-seq was undertaken at Wuhan Seqhealth Technology Co., Ltd. (Wuhan, China).

\section{Immunofluorescence staining}

Cells were seeded on coverslips and incubated with antibody specific for p113 (ABclonal Biotechnology Co., Ltd), Flag-tag (14793S, Cell Signaling Technology Inc.), 4-hydroxynonenal (4-HNE, MAB3249, Novus Biologicals, Ltd., Centennial, CO), or ALDH3A1 (ab186726, Abcam Inc.) at room temperature for $2 \mathrm{~h}$. Then, coverslips were incubated with Alexa Fluor 594 goat anti-mouse IgG or Alexa Fluor 488 goat anti-rabbit IgG, and stained with 4',6-diamidino-2-phenylindole (DAPI, $300 \mathrm{nmol} \cdot \mathrm{L}^{-1}$, Sigma).

\section{Bimolecular fluorescence complementation (BiFC) assay} Human $p 113$ ORF (342bp), ZRF1 cDNA (1866bp), and $B R D 4$ cDNA (4089bp) were subcloned into pBiFCVN173 or pBiFC-VC155 (Addgene), and co-transfected into tumor cells with Lipofectamine 2000 (Invitrogen) for $24 \mathrm{~h}$. The fluorescence was observed with a confocal microscope (Nikon, Japan) $[15,16,19]$.

\section{Design and synthesis of inhibitory peptides}

Inhibitory peptides for blocking interaction between p113 and ZRF1 were designed. The 11-amino acid long peptide (YGRKKRRQRRR) from Tat protein transduction domain served as a cell-penetrating peptide. Thus, inhibitory peptides were chemically synthesized by linking with $\mathrm{N}$-terminal biotin-labeled cell-penetrating peptide and C-terminal fluorescein isothiocyanate (FITC, ChinaPeptides Co. Ltd., Shanghai, China), with purity larger than $95 \%$.

\section{Biotin-labeled peptide pull-down assay}

Cellular proteins were isolated using $1 \times$ cell lysis buffer (Promega), and incubated with biotin-labeled peptide and streptavidin-agarose at $4{ }^{\circ} \mathrm{C}$ for $2 \mathrm{~h}$. Beads were extensively washed, and protein pulled down was measured by western blot.

\section{Sucrose gradient sedimentation}

Sedimentation of polysomal fractions was performed as previously described [20]. Briefly, tumor cells were treated with $100 \mu \mathrm{g} / \mathrm{ml}$ of cycloheximide (Sigma) for 5-10 min. Cell extracts were layered on top of $15-30 \%(\mathrm{w} / \mathrm{v})$ linear sucrose gradient and centrifugated $(40,000 \times \mathrm{g})$ at $4{ }^{\circ} \mathrm{C}$ for $2 \mathrm{~h}$. Polysome-bound transcripts in collected gradient fractions were detected by real-time qRT-PCR.

\section{Lipid profiling assay}

Target metabolic profiling was performed by gas chromatography-mass spectrometry (GC-MS) at Wuhan Metware Biotechnology Co, Ltd. (Wuhan, China). Briefly, samples were quickly frozen in liquid nitrogen, ultrasonically crushed, and filtered through SPE column. Then, SPE column was extracted with $\mathrm{n}$-hexane and detected by GC-MS.

\section{Seahorse extracellular flux assay}

Cells were seeded in XF cell culture microplates $(30,000$ / well) at $37^{\circ} \mathrm{C}$ for $24 \mathrm{~h}$, cultured in FAO assay medium (pH 7.4, $2.5 \mathrm{mmol} \cdot \mathrm{L}^{-1}$ glucose, $0.5 \mathrm{mmol} \cdot \mathrm{L}^{-1}$ carnitine) without $\mathrm{CO}_{2}$ for $1 \mathrm{~h}$, and incubated with bovine serum albumin (BSA) or oleic acid-BSA $\left(200 \mu \mathrm{mol} \cdot \mathrm{L}^{-1}\right.$, Sigma). Then, oligomycin $\left(1 \mu \mathrm{mol} \cdot \mathrm{L}^{-1}\right)$, carbonyl cyanide-p-trifluoromethoxyphenylhydrazone (FCCP, $2 \mu \mathrm{mol} \cdot \mathrm{L}^{-1}$ ), and rotenone/antimycin A $\left(0.5 \mu \mathrm{mol} \cdot \mathrm{L}^{-1}\right.$, Sigma $)$ were added, while oxygen consumption rate (OCR) was detected using a Seahorse Biosciences XFe24 Flux Analyzer (North Billerica, MA).

\section{Mitochondrial structure, membrane potential $(\Delta \psi \mathrm{m})$, and complex I activity assays}

For imaging mitochondrial mass or morphology independent of membrane potential [21], live cells were stained by

\footnotetext{
(See figure on next page.)

Fig. 1 Serum deprivation stress promotes expression of p113 derived from a circRNA of CUX1 in NB. a Heatmap of proteomics (left panel) and Venn diagram (right panel) showing altered proteins in SH-SY5Y and SK-N-BE(2) cells treated by serum deprivation (SD) for 24 h, and overlapping analysis with established transcription factors derived from Genomatrix database (http://www.genomatix.de). b Western blot assay indicating the expression of CUX1 isoforms and TCF3 in SH-SY5Y and SK-N-BE(2) treated with SD as indicated. c Mass spectrometry assay revealing amino acid sequence of $\mathrm{p} 113$ recovered from electrophoresis gel, with additional amino acids produced by ecircCUX1 (red). $\mathbf{d}$ Schematic illustration showing the genomic location of ecircCUX1 generated from exons 9-11 of CUX1, and validation by RT-PCR using convergent or divergent primers and Sanger sequencing. e Western blot assay (right panel) indicating endogenous and exogenous expression of p113 in SH-SY5Y cells stably transfected with empty vector (mock), wild-type or mutant 3Flag-tagged ecircCUX1 as indicated (left panel). f Western blot assay showing the levels of p113 and CUX1 isoforms in SH-SY5Y cells stably transfected with mock or ecircCUX1, and those treated with or without SD for $24 \mathrm{~h}$. $\mathbf{g}$ Schematic illustration indicating genomic knockin (KI) of 3Flag-tagged or mutant (Mut) form of ecircCUX1 using CRISPR-Cas9. $\mathbf{h}$ Western blot assay revealing endogenous and exogenous expression of p1 13 in HEK293T and SK-N-BE(2) cells with genomic knockin (KI) of 3Flag-tagged or mutant (Mut) form of ecircCUX1. Fisher's exact test for overlapping analysis in $\mathbf{a}$. Data are representative of three independent experiments in $\mathbf{b}, \mathbf{d}-\mathbf{f}$ and $\mathbf{h}$
} 


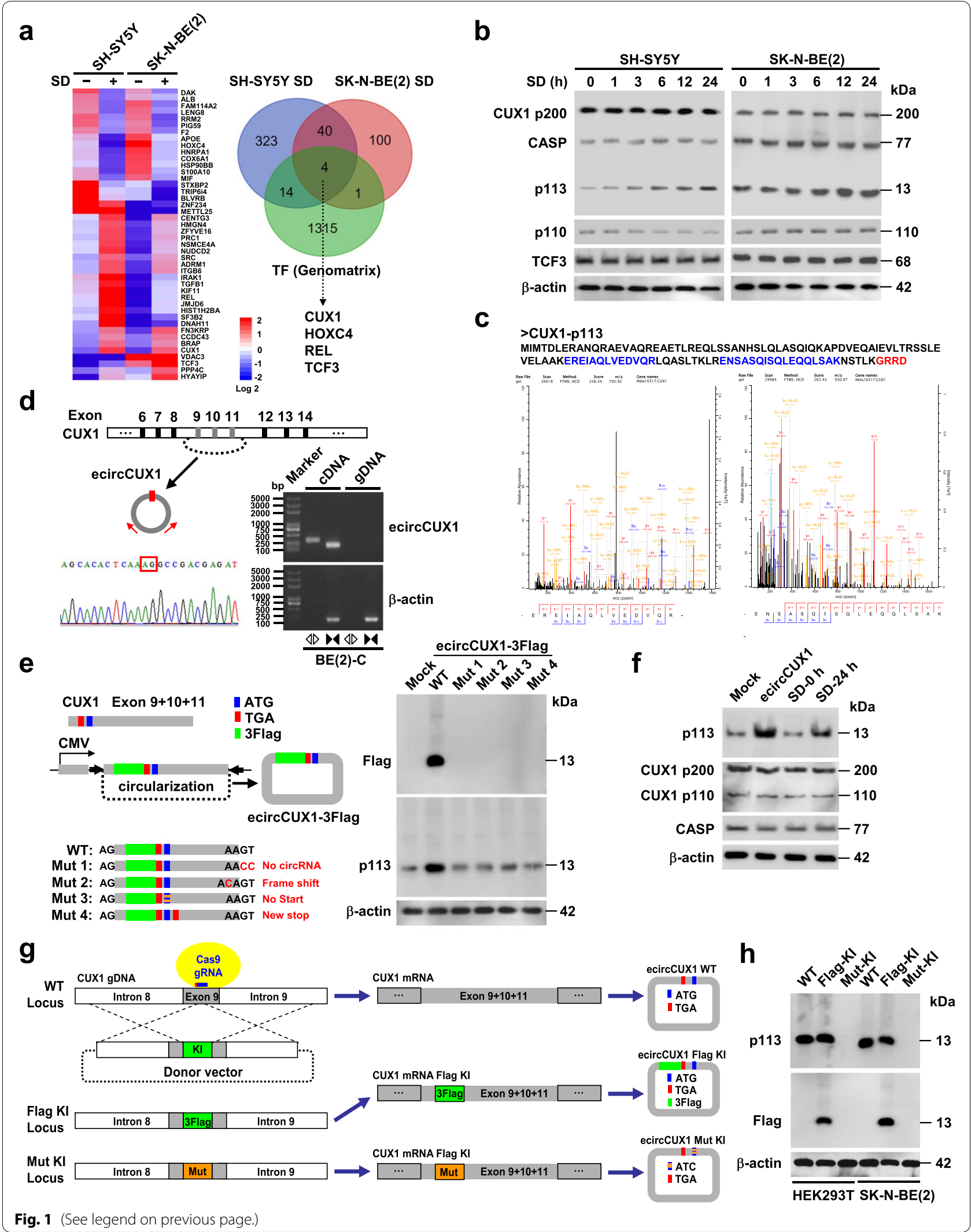


MitoTracker Green $\left(100 \mathrm{nmol} \cdot \mathrm{L}^{-1}\right.$, Invitrogen) at $37^{\circ} \mathrm{C}$ for $30 \mathrm{~min}$ or transfected with MitoRFP reporter (Addgene) for $48 \mathrm{~h}$, with nuclei staining by Hochest 33,342 (Sigma). Mitochondrial structure was observed by transmission electron microscopy [22]. Cells were treated with JC-1 $(10 \mu \mathrm{g} / \mathrm{ml}$, Sigma) at $37^{\circ} \mathrm{C}$ for $20 \mathrm{~min}$, while red fluorescence intensity reflecting $\Delta \psi \mathrm{m}$ was assessed with a fluorescence microscope. Mitochondrial complex I activity was determined by Mitochondrial Complex I Activity Assay Kit (Sigma).

\section{$\mathrm{NAD}^{+} / \mathrm{NADH}$ ratio and ATP level measurement}

Cellular nicotinamide adenine dinucleotide $\left(\mathrm{NAD}^{+}\right) /$ nicotinamide adenine dinucleotide $(\mathrm{NADH})$ ratio or ATP levels were measured using $\mathrm{NAD}^{+} / \mathrm{NADH}$ Assay Kit (ab65348, Abcam Inc.) and ATP Determination Kit (A22066, Invitrogen), respectively.

\section{In vitro cell viability, growth, and invasion assays}

In vitro viability, growth, and invasive capabilities of tumor cells were detected by 2-(4,5-dimethyltriazol-2-yl)-2,5-diphenyl tetrazolium bromide (MTT; Sigma) colorimetric [23, 24], soft agar $[15,16,19]$, and matrigel invasion $[15,16,19]$ assays.

\section{In vivo tumorigenesis and aggressiveness assays}

Four-week-old male BALB/c nude mice (National Rodent Seeds Center, Shanghai, China) were reared at specific pathogen free condition. For in vivo tumor growth, metastasis, and peptide therapeutic studies, tumor cells $\left(1 \times 10^{6}\right.$ or $0.4 \times 10^{6}$ ) were injected into dorsal flanks or tail vein of blindly randomized nude mice ( $n=5$ per group), respectively. For therapeutic studies, 1 week after injection, mice were blindly randomized and treated by tail vein injection of synthesized cell-penetrating peptides (ChinaPeptides, Shanghai, China) as indicated $[15,16,19]$.

\section{Human tissue samples}

All procedures were conformed to principles set forth by Declaration of Helsinki. Written informed consent was obtained from all legal guardians of patients without a history of preoperative chemotherapy or radiotherapy. Human normal dorsal root ganglia were collected from therapeutic abortion. Fresh tumor tissues were collected at surgery, validated by pathological diagnosis, and stored at $-80^{\circ} \mathrm{C}$.

\section{Immunohistochemistry}

Immunohistochemical staining and quantitative evaluation were performed as previously described $[15,16,19]$, with antibody specific for p113 (ABclonal Biotechnology Co.), Ki-67 (ab92742, Abcam Inc.), or CD31 (ab28364, Abcam Inc.).

\section{Data and code availability}

RNA-seq and ChIP-seq data have been deposited in Gene Expression Omnibus (GEO) database (https://www.ncbi. nlm.nih.gov/geo/, accession number GSE182329 and GSE182402). Public datasets are available from GEO database (GSE62564, GSE25066, GSE17679, GSE65904, GSE2658) or The Cancer Genome Atlas (TCGA) database (https://cancergenome.nih.gov).

\section{Statistical analysis}

All data were shown as mean \pm standard error of the mean (s.e.m.). Cutoff values were determined by average gene expression levels. Student's $t$ test, analysis of variance (ANOVA), and $\chi^{2}$ analysis were applied to compare difference. Fisher's exact test was applied to analyze statistical significance of overlapping. Log-rank test was used to assess survival difference. All statistical tests were two-sided and considered significant when false discovery rate (FDR)-corrected $P$ values were less than 0.05 .

\section{Results \\ Serum deprivation stress promotes expression of $\mathrm{p} 113$ derived from a circRNA of $C U X 1$ in NB}

To investigate crucial factors regulating metabolic reprogramming, NB cell lines were treated by serum

\footnotetext{
(See figure on next page.)

Fig. 2 p113 is upregulated and facilitates fatty acid oxidation and mitochondrial activity in NB. a Western blot assay showing the p113 levels in normal dorsal ganglia (DG), tumor (T) tissues of NB, and cultured tumor cell lines. $\mathbf{b}$ Western blot assay indicating the expression of p1 13 and CUX1 isoforms (p200 and p110) in SH-SY5Y, SH-N-SH, BE(2)-C, and IMR32 cells stably transfected with empty vector (mock), ecircCUX1, ecircCUX1 with ORF mutation (ecircCUX1 Mut), scramble shRNA (sh-Scb), or sh-ecircCUX1. c Western blot assay showing the cytoplasmic and nuclear accumulation of p113 in SH-SY5Y cells stably transfected with mock or p113. d Immunofluorescence assay revealing cytoplasmic and nuclear localization of p113 in SH-SY5Y and SK-N-SH cells stably transfected with Flag-tagged p113 (upper panel), and those in BE(2)-C and IMR32 cells (lower panel), with nuclei stained by DAPI (blue). Scale bar: $10 \mu \mathrm{m}$. e and $\mathbf{f}$ Heatmap (e) and quantification (f) of metabolite profiling assay indicating the fatty acid levels in SH-SY5Y and BE(2)-C cells stably transfected with mock, ecircCUX1, ecircCUX1 Mut, p113, sh-Scb, or sh-ecircCUX1 $(n=3)$. g Seahorse extracellular flux assay showing the oxygen consumption rate (OCR) levels in SH-SY5Y and BE(2)-C cells stably transfected with mock, ecircCUX1, ecircCUX1 Mut, p113, sh-Scb, or sh-ecircCUX1, and those treated with BSA or oleic acid $\left(\mathrm{OLE}, 200 \mu \mathrm{mol} \cdot \mathrm{L}^{-1}, n=4\right)$. $\mathbf{h}$ Relative NAD ${ }^{+}$/NADH ratio and ATP levels in SH-SY5Y and BE(2)-C cells stably transfected with mock, ecircCUX1, ecircCUX1 Mut, p113, sh-Scb, or sh-ecircCUX1, and those treated with BSA or OLE $\left(200 \mu \mathrm{mol} \cdot \mathrm{L}^{-1}, n=5\right)$. ANOVA compared the difference in $\mathbf{f}-\mathbf{h} .{ }^{*} P<0.05 \mathrm{vs}$. mock, sh-Scb, or sh-Scb + BSA. Data are shown as mean \pm s.e.m. (error bars) and representative of three independent experiments in $\mathbf{a}-\mathbf{d}, \mathbf{g}$ and $\mathbf{h}$
} 
a

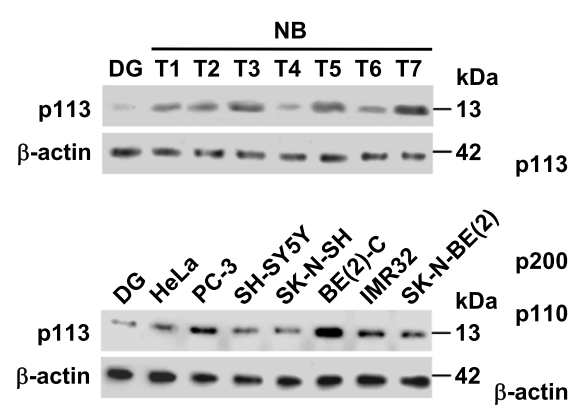

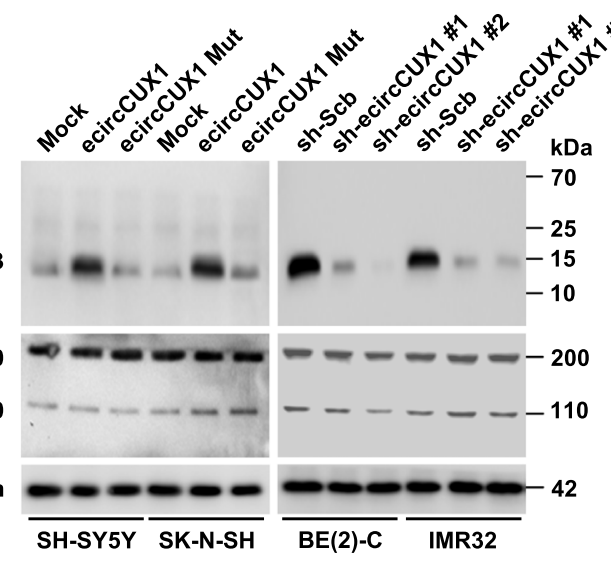

\section{C}

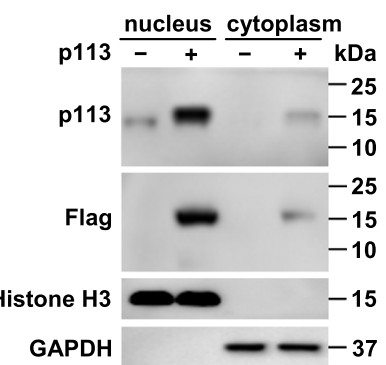

d

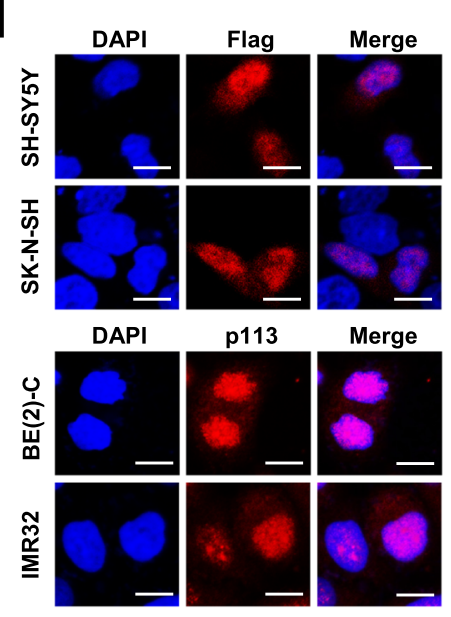

g
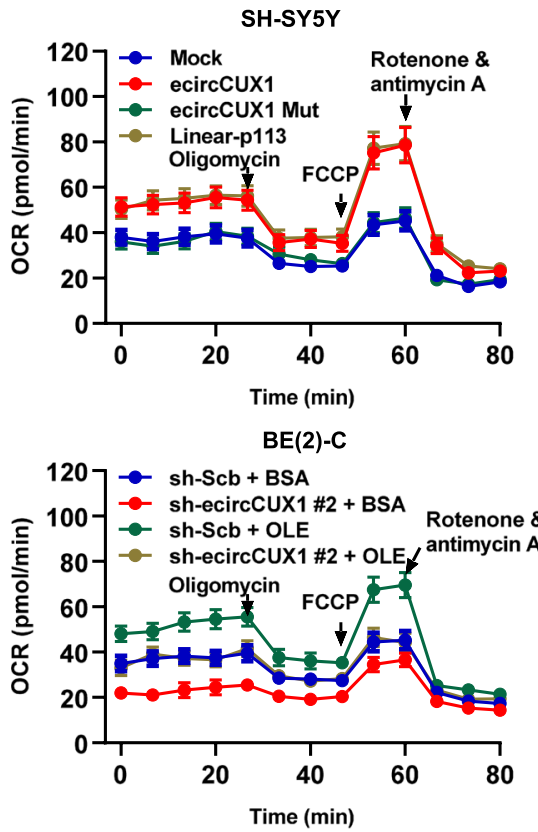

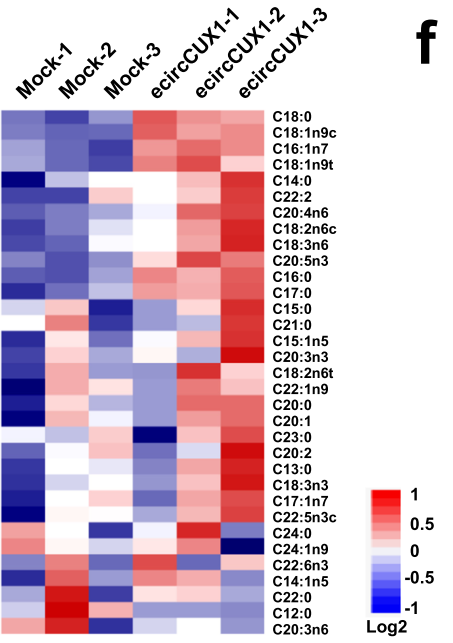

h
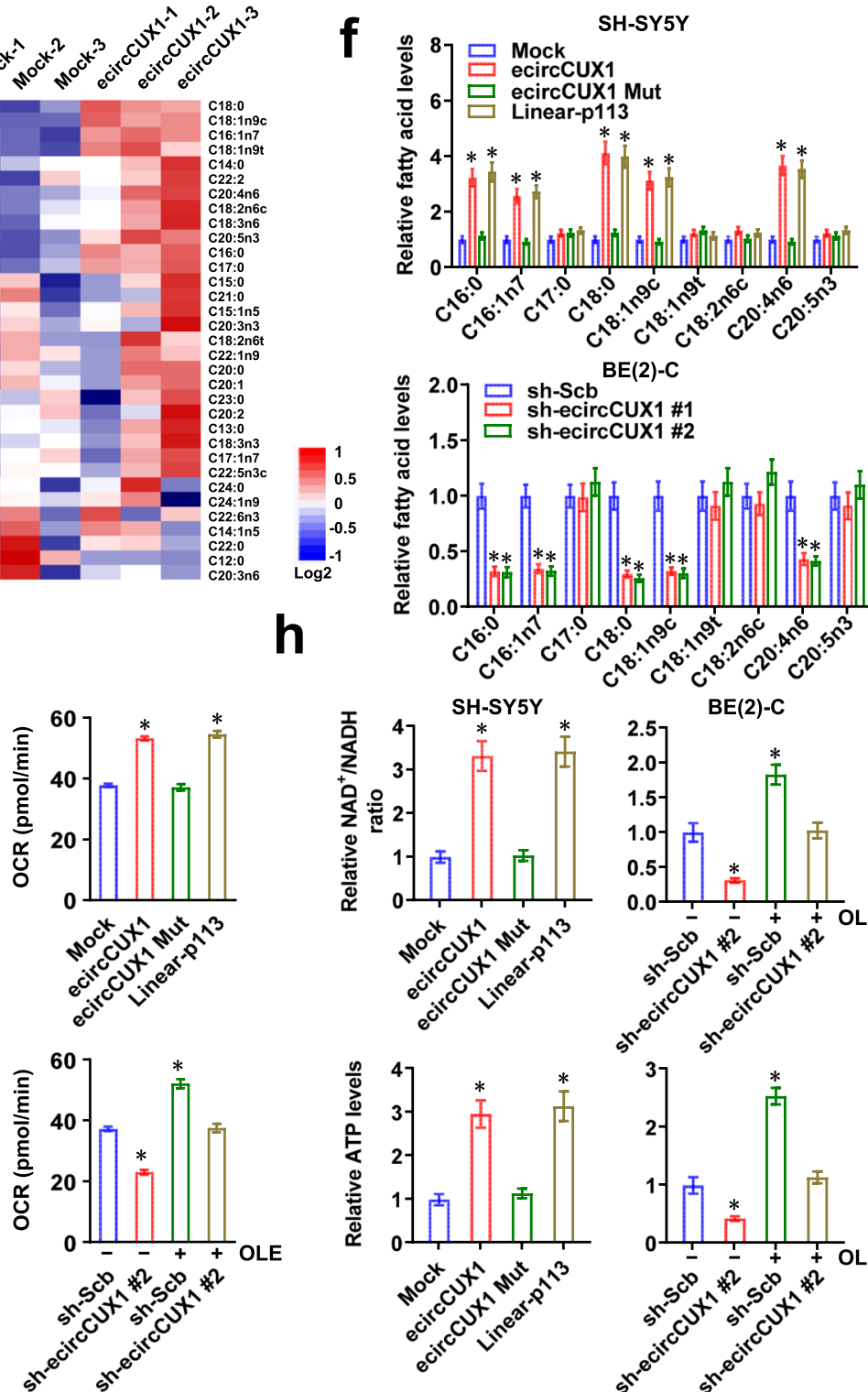

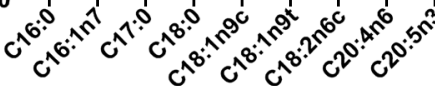
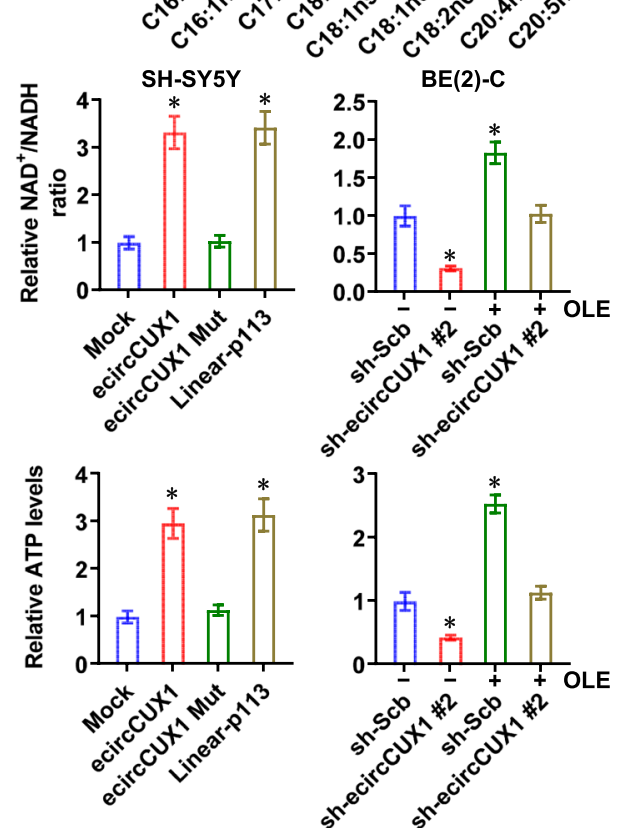

Fig. 2 (See legend on previous page.) 
deprivation (SD), a condition causing metabolic stress [25], while altered proteins were analyzed by isobaric tags for relative and absolute quantification (iTRAQ). There were 381 and 145 differentially expressed proteins in SH-SY5Y and SK-N-BE(2) cells upon SD for $24 \mathrm{~h}$, respectively (Additional file 1: Table S4), and overlapping with Genomatix database (http://www.genom atix.de/) indicated that expression of four transcription factors was significantly altered (Fig. 1a). Among them, CUT-like homeobox 1 (CUX1) and TCF3 were consistently upregulated in both NB cell lines. Validating western blot assay indicated that expression of major CUX1 isoforms [p200, p110, or CDP/CUT alternatively spliced product (CASP)] and TCF3 remained unaffected, while a new CUX1 isoform with a molecular weight of $13 \mathrm{kDa}$ was unexpectedly upregulated in a time-dependent manner upon SD treatment (Fig. 1b). The amino acid sequence of this new isoform was deduced from exons 10 and 11 of CUX1 (Fig. 1c). Analysis from circRNADb database (http://reprod.njmu.edu.cn/circrnadb) identified a spanning junction ORF-containing circRNA (hsa_circ_30402) derived from exons 9-11 of CUX1 (referred as ecircCUX1), which might encode a conserved 113-amino acid protein (p113) in primates (Fig. 1d and Additional file 1: Fig. S1a). Accurate circularization of ecircCUX1 was validated in $\mathrm{BE}(2)-\mathrm{C}$ cells (Fig. 1a), with cytoplasmic abundance (Additional file 1: Fig. S1b). Notably, SD treatment did not affect the ecircCUX1 levels (Additional file 1: Fig. S1c), but increased their binding to polysomes in NB cells (Additional file 1: Fig. S1d). Using prepared p113 antibody or Flagtag antibody, western blot assay revealed the increase of p113 levels in NB cells transfected with Flag-tagged wild-type ecircCUX1, but not with ORF mutant vector (Fig. 1e). In addition, ectopic expression of ecircCUX1 or SD treatment led to upregulation of p113, but not of p200, p110 or CASP, in NB cells (Fig. 1f). Next,
CRISPR-Cas9-mediated knockin of Flag-tagged ecircCUX1, but not of mutant ecircCUX1, resulted in p113 expression in HEK293T and SK-N-BE(2) cells (Fig. 1g, $\mathrm{h}$ and Additional file 1: Fig. S1e, f). Importantly, IRES of ecircCUX1 induced a remarkable luciferase activity (Additional file 1: Fig. S1g), indicating its $5^{\prime}$ cap-independent translation pattern. These results demonstrated that SD stress promoted the expression of p113 derived from a circRNA of $C U X 1$ in NB cells.

\section{p113 is upregulated and facilitates fatty acid oxidation and mitochondrial activity in NB}

Then, we observed the expression profiles of p113 in tumor tissues and cells. Higher p113 levels were noted in NB specimens and cell lines, than those of normal dorsal root ganglia (Fig. 2a). Endogenous p113 expression was noted in nuclei of tumor cells (Additional file 1: Fig. S2a), which was detected in 28/42 (66.7\%) NB cases, with higher expression in those with elder age $(P=0.001)$, poor differentiation $(P=0.001)$, higher mitosis karyorrhexis index $(P=0.002)$, or advanced international neuroblastoma staging system (INSS) stages $(P=0.003$, Additional file 1: Table S5). In these patients, high p113 expression was associated with poor survival probability $\left(P<1.0 \times 10^{-4}\right.$, Additional file 1 : Fig. S2b). Elevated p113 expression was also detected in a variety of cancer tissues (Additional file 1: Fig. S2c). Using SH-SY5Y, SK-N-SH, BE(2)-C, and IMR32 cells (with low or high p113 levels) as models, stable transfection of wild-type ecircCUX1 or two independent shRNAs targeting junction site of ecircCUX1 (sh-ecircCUX1) resulted in over-expression or silencing of $p 113$, but not of $p 200$ or $p 110$ (Fig. 2b and Additional file 1: Fig. S2d), mainly localizing at nuclei (Fig. 2c, d). No alteration of p113 expression was observed in NB cells stably transfected with ORF mutant form of ecircCUX1 (Fig. 2b).

\footnotetext{
(See figure on next page.)

Fig. 3 p113 physically interacts with ZRF1 and BRD4 in NB cells. a Volcano plots showing differentially expressed genes (fold change> 1.5, $P<0.05$ ) in SH-SY5Y cells stably transfected with empty vector (mock) or ecircCUX1. b Coomassie blue staining (left panel) and Venn diagram (right panel) revealing identification of p113-interacting proteins pulled down by p113 or Flag-tag antibody in SH-SY5Y cells stably transfected with 3Flag-tagged $p 113$, and those overlapped with transcription factors (TF) or epigenetic factors derived from ChIP-X and EpiFactors databases. c Co-IP and western blot assays indicating the interaction among p113, ZRF1, and BRD4 in SH-SY5Y and BE(2)-C cells stably transfected with mock, ecircCUX1, scramble shRNA (sh-Scb), or sh-ecircCUX1. d Secondary co-IP assays showing protein interaction among P113, ZRF1, and BRD4 in SH-SY5Y cells stably transfected with HA-tagged p113, Flag-tagged ZRF1, and His-tagged BRD4. e BiFC assay revealing the interaction of p113 with ZRF1 or BRD4 (arrowheads) in SH-SY5Y cells stably transfected with indicated constructs, with nuclei stained by DAPI. Scale bars: $10 \mu \mathrm{m}$. $\mathbf{f}$ and g Western blot assay (g) validating the knockdown of ZRF1 or BRD4 in SH-SY5Y cells stably transfected with scramble (Scb) or specific sgRNA for CRISPR interference (CRISPRi, f). Wild type (WT) cells were taken as negative controls. $\mathbf{h}$ Co-IP and western blot assays indicating the interaction of p1 13 with ZRF1 or BRD4 in SH-SY5Y cells stably transfected with mock or ecircCUX1, and those co-transfected with CRISPRi sgRNA specific against ZRF1 or BRD4. i Schematic illustration of protein interaction among p113, ZRF1, and BRD4. j Dual-luciferase assay showing the activity of ZRF1 in NB cells stably transfected with mock, ecircCUX1, ecircCUX1 Mut, $p 113$, sh-Scb, or sh-ecircCUX1 $(n=5)$. Fisher's exact test for overlapping analysis in $\mathbf{b}$. ANOVA compared the difference in $\mathbf{j}$. ${ }^{*} P<0.05 \mathrm{vs}$. mock or sh-Scb. Data are shown as mean \pm s.e.m. (error bars) and representative of three independent experiments in $\mathbf{c}-\mathbf{e}, \mathbf{g}, \mathbf{h}$ and $\mathbf{j}$
} 
a

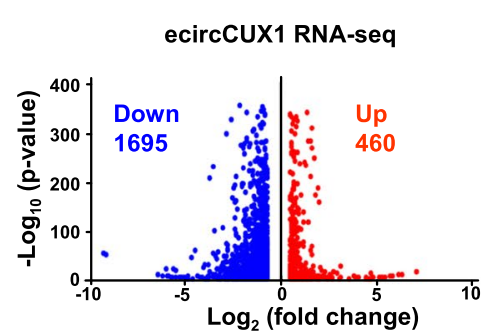

C

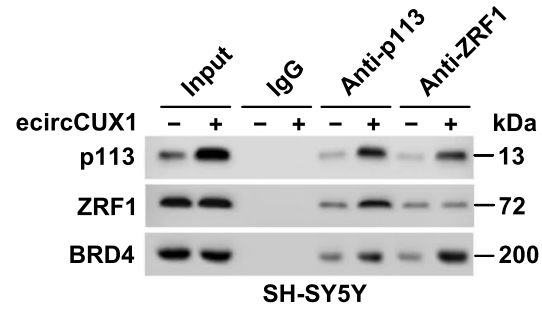

d

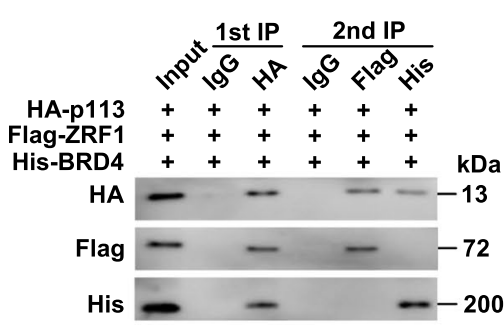

b

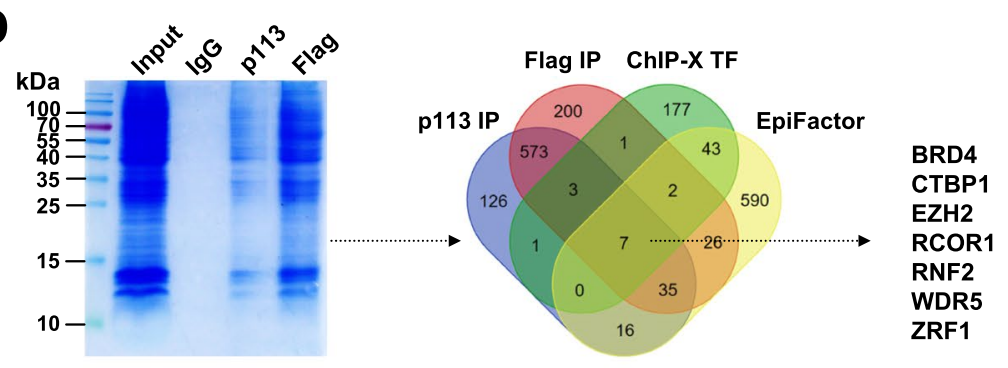

f

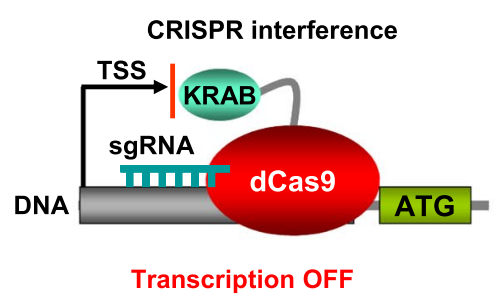

g

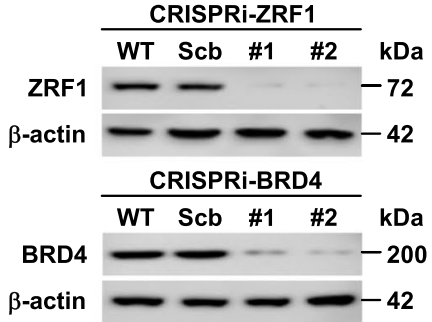

e

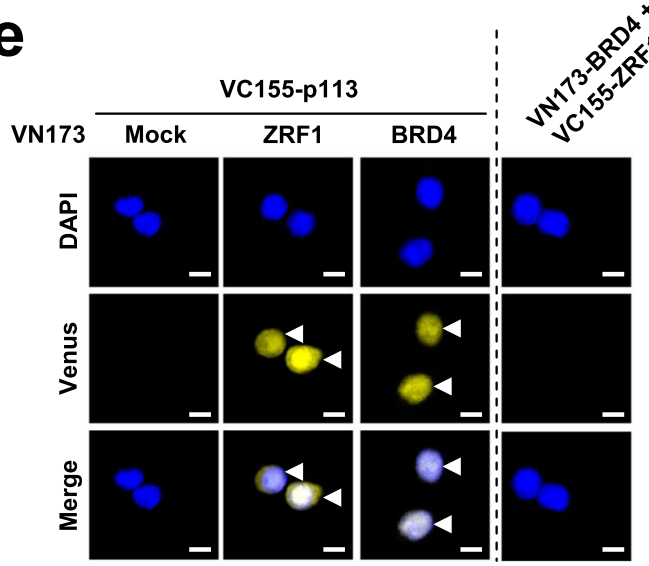

i

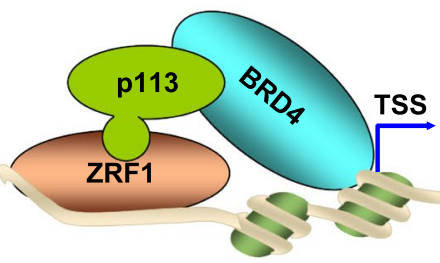

h
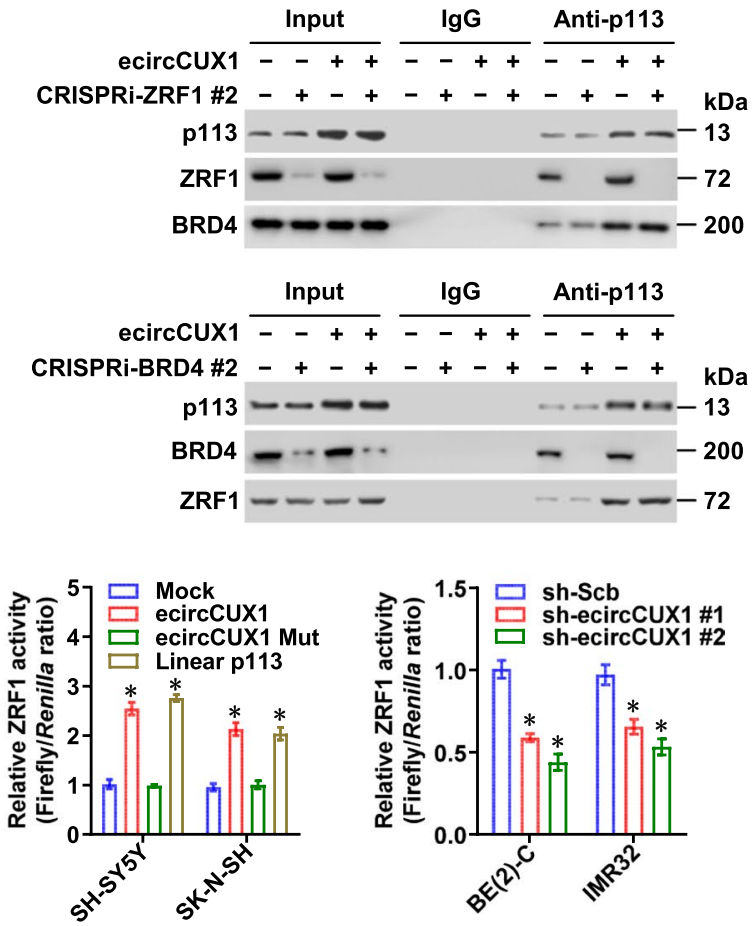

Fig. 3 (See legend on previous page.) 
Since previous studies implicate the potential impacts of SD on lipid metabolic reprogramming [26], lipid metabolite profiling assay was further undertaken, which revealed the increase of long-chain fatty acid production in SH-SY5Y cells stably transfected with ecircCUX1 (Fig. 2e). Of note, over-expression of ecircCUX1 or $p 113$, but not of ecircCUX1 with ORF mutation (ecircCUX1 Mut), increased the levels of palmitic acid (C16:0), palmitoleic acid (C16:1n7), oleic acid (C18:1n9c), stearic acid (C18:0), and arachidonic acid (C20:4n6), while knockdown of ecircCUX1 exerted the opposite effects (Fig. 2f). To explore the potential roles of ecircCUX1 in FAO, NB cells were treated with oleic acid under fatty acid-restricted conditions. There was elevated OCR, mitochondrial membrane potential, complex I activity, $\mathrm{NAD}^{+} / \mathrm{NADH}$ ratio, and ATP production in SH-SY5Y and $\mathrm{BE}(2)-\mathrm{C}$ cells stably transfected with ecircCUX1 or p113, but not with ecircCUX1 Mut (Fig. $2 \mathrm{~g}$, h and Additional file 1: Fig. S2e, f). Meanwhile, silencing of ecircCUX1 abolished the increase of OCR, mitochondrial membrane potential, complex I activity, $\mathrm{NAD}^{+} / \mathrm{NADH}$ ratio, and ATP production induced by exogenous oleic acid (Fig. 2g, h and Additional file 1: Fig. S2e, f). However, ectopic expression of ecircCUX1 or p113 did not affect mitochondrial mass in NB cells, with intact twolayer membrane and inner structures (Additional file 1: Fig. S3a-c). These results suggested that p113 was upregulated and facilitated fatty acid oxidation and mitochondrial activity in NB.

ecircCUX1 promotes growth and aggressiveness of NB cells via encoding $\mathbf{p} 113$

We further explored the biological impacts of ecirc$C U X 1$ on growth and aggressiveness of NB. Stable over-expression of ecircCUX1 or p113, but not of ecircCUX1 Mut, facilitated the growth and invasion capability of SH-SY5Y and SK-N-SH cells (Additional file 1: Fig. S4a-d). Meanwhile, the growth and invasion of BE(2)-C and IMR32 cells were significantly reduced by knockdown of ecircCUX1 (Additional file 1: Fig. S4a-d). Consistently, stable transfection of ecircCUX1 or sh-ecircCUX1 \#2 into NB cells resulted in a significant increase or decrease of growth, tumor weight, long-chain fatty acid levels, complex I activity, $\mathrm{NAD}^{+} / \mathrm{NADH}$ ratio, ATP production, Ki-67 proliferation index, and CD31-positive microvessels of subcutaneous xenografts in athymic nude mice (Additional file 1: Fig. S4e, S5a, S5b). In experimental metastasis assay, athymic nude mice treated with tail vein injection of SH-SY5Y or BE(2)-C cells stably transfected with ecircCUX1 or sh-ecircCUX1 \#2 displayed more or fewer lung metastatic colonies, with lower or greater survival probability, respectively (Additional file 1: Fig. S5c, d). These results suggested that ecircCUX1 promoted the growth and aggressiveness of NB cells via encoding $\mathrm{p} 113$.

\section{p113 physically interacts with ZRF1 and BRD4 in NB cells}

To characterize downstream targets of p113, RNA-seq assay was performed, which indicated 460 upregulated and 1695 downregulated genes in SH-SY5Y cells upon ecircCUX1 over-expression (Fig. 3a). Co-IP and subsequent mass spectrometry assays revealed 761 and 847 protein pulled down by p113 or Flag antibody in SH-SY5Y cells stably transfected with 3Flag-tagged p113 (Additional file 1: Table S6 and Table S7), and overlapping analysis with transcription factors and epigenetic factors derived from ChIP-X program [27] and EpiFactors database [28] identified seven potential p113-interacting proteins (Fig. 3b). Among them, ZRF1 was the only transcription factor, while BRD4 might serve as a transcriptional co-factor. Endogenous interaction of p113 with ZRF1 and BRD4 was observed in NB cells, which was respectively increased or decreased by ectopic expression or knockdown of ecircCUX1, but not affected by transfection of ecircCUX1 with ORF mutation (Fig. 3c and Additional file 1: Fig. S6a). Two-step immunoprecipitation assay revealed respective binding of p113 to ZRF1 or BRD4 (Fig. 3d). Especially, SANT domain (450-621 aa) of

\footnotetext{
(See figure on next page.)

Fig. 4 p113/ZRF1/BRD4 complex promotes lipid metabolic reprogramming and mitochondrial complex I activity in NB cells. a Heatmap, distribution, and binding motif of ChIP-Seq (left panel) assay revealing genomic enrichment of ZRF1 in SH-SY5Y cells, while Venn diagram, heatmap, and GO pathway (right panel) showing identification of p113/ZRF1/BRD4 target genes by overlapping analysis of RNA-seq results upon p113 over-expression and ChIP-seq peaks of ZRF1 or BRD4. b ChIP-seq assay showing the binding peak of BRD4 or ZRF1 on promoter regions of ALDH3A1, NDUFA1, or NDUFAF5 in SH-SY5Y cells. c Western blot assay indicating the expression of ALDH3A1, NDUFA1, or NDUFAF5 in SH-SY5Y cells stably transfected with empty vector (mock) or ecircCUX1, and those co-transfected with sgRNA specific against ZRF1 or BRD4 for CRISPRi. d Schematic illustration showing the involvement of ALDH3A1, NDUFA1, or NDUFAF5 in lipid metabolic reprogramming and mitochondrial respiratory activity. e Relative OCR levels in SH-SY5Y cells stably transfected with mock or ecircCUX1, and those co-transfected with sgRNA specific against ZRF1 or BRD4 for CRISPRi $(n=5)$ ). f Relative fatty acid levels, complex I activity, NAD ${ }^{+} / N A D H$ ratio, and ATP levels in SH-SY5Y cells stably transfected with mock or ecirCCUX1, and those co-transfected with sgRNA specific against ZRF1 or BRD4 for CRISPRi $(n=5)$. ANOVA compared the difference in e and $\mathbf{f}$. ${ }^{*} P<0.05$ vs. mock+CRISPRi-Scb. Data are shown as mean \pm s.e.m. (error bars) and representative of three independent experiments in $\mathbf{c}$, e and $\mathbf{f}$
} 
a
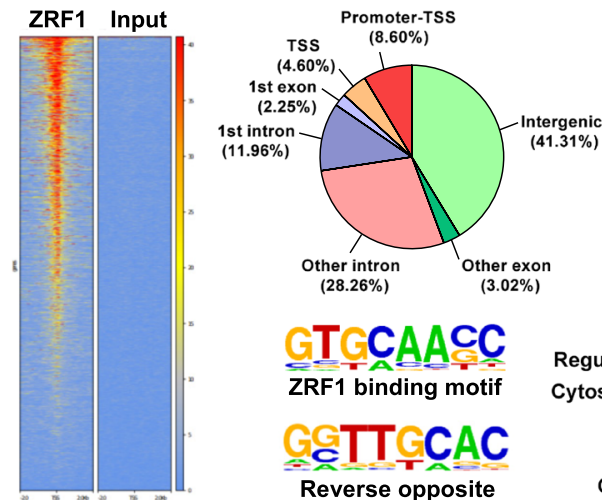

GTCAACC ZRF1 binding motif

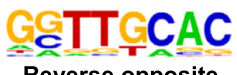

$$
\text { Rytos }
$$

Regulation of cell division-

ytoskeleton organization Cell-cell signaling

Metabolic pathways Reverse opposite Complex I biogenesis
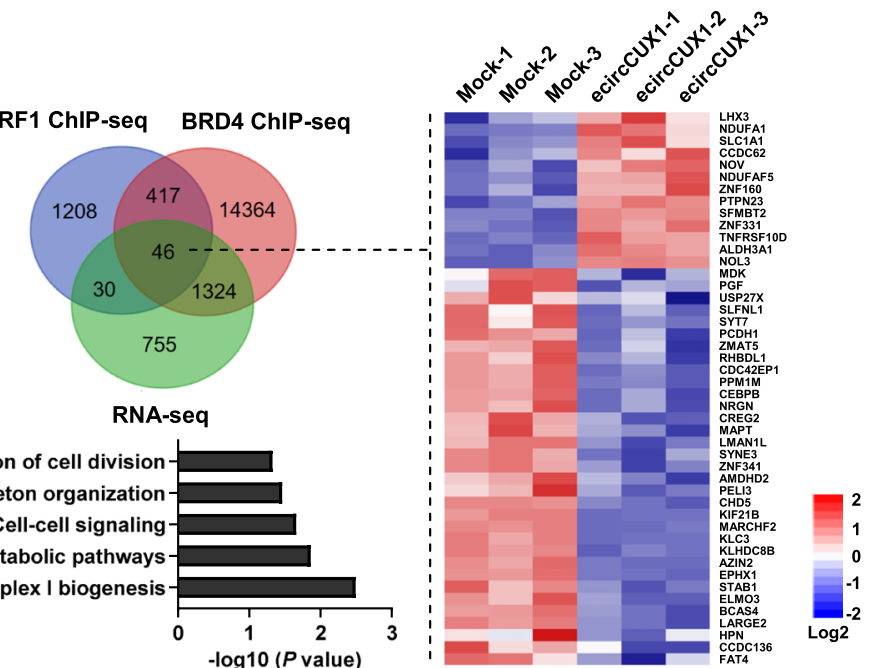

b

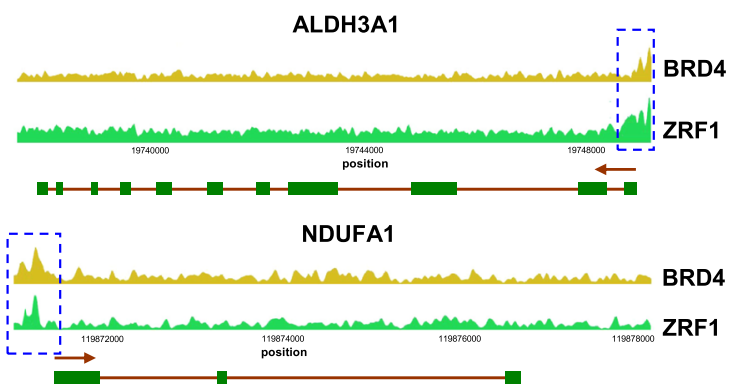

C

CRISPRi-ZRF1 \#2 - - + + ecircCUX1 - $+-+k$

$\mathrm{p} 113--13$

ZRF1 --72

ALDH3A1 - -55

NDUFA1 - -14

NDUFAF5 - - - 39

$\beta$-actin ---42

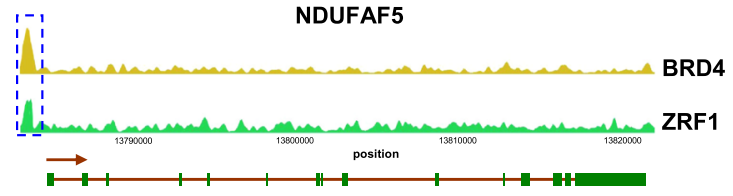

d

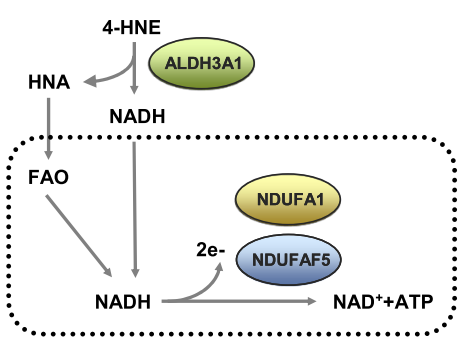

e

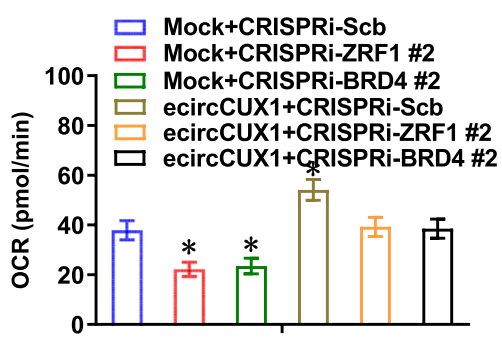

CRISPRI-BRD4 \#2 - $\quad-\quad+\quad+$

ecircCUX1 - + - + $k D a$

p113 --13

BRD4 -200

ALDH3A1 $-\square 5$

NDUFA1 - - -14

NDUFAF5 - - -39

$\beta$-actin

\section{f}

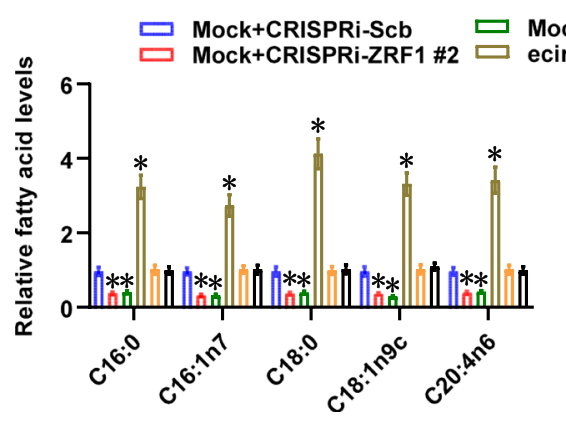

ock+CRISPRi-BRD4 \#2 $\square$ ecircCUX1+CRISPRi-ZRF1 \#2
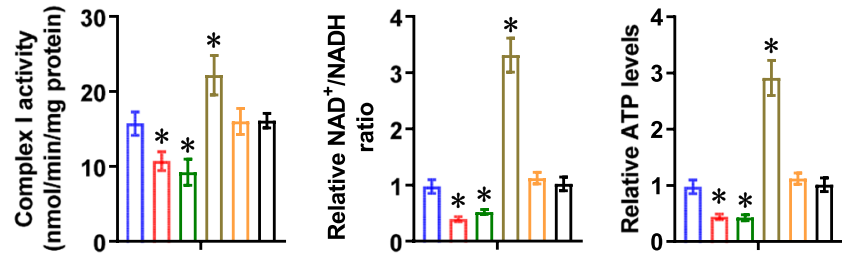

Fig. 4 (See legend on previous page.) 
ZRF1 as well as bromodomain 2 (BD2) domain (250470 aa) of BRD4 were essential for their interaction with p113 (Additional file 1: Fig. S6b-d). In BiFC assay [29], p113 physically interacted with ZRF1 and BRD4, whereas no interaction was noted between ZRF1 and BRD4 (Fig. 3e). Then, CRISPR interference (CRISPRi) approach [30] was applied for knockdown of ZRF1 or BRD4 (Fig. 3f, g). Ectopic expression of ecircCUX1 increased the interaction of p113 with ZRF1 or BRD4, which was individually abolished by silencing of $Z R F 1$ or BRD4, respectively (Fig. 3h), indicating the mediating roles of p113 in forming trimer complex with ZRF1 and BRD4 (Fig. 3i). Notably, ectopic expression or knockdown of ecircCUX1 facilitated and attenuated the transactivation of ZRF1 in NB cells, respectively (Fig. 3j). Meanwhile, ectopic expression of ecircCUX1 with ORF mutation did not affect transcriptional activity of ZRF1 (Fig. 3j). These results indicated that p113 physically interacted with ZRF1 and BRD4 as a trimer complex in NB cells.

\section{p113/ZRF1/BRD4 complex promotes lipid metabolic reprogramming and mitochondrial complex I activity in NB cells}

To further investigate target genes of p113/ZRF1/ BRD4 complex, ChIP-seq assay was performed using ZRF1- or BRD4-specific antibody, while results were overlappingly analyzed with ecircCUX-regulated genes derived from RNA-seq. Comprehensive analysis revealed 46 target genes regulated by transcriptional trimer complex p113/ZRF1/BRD4, especially those involved in metabolic pathway or complex I biogenesis, including $A L D H 3 A 1, N D U F A 1$, and NDUFAF5 (Fig. 4a, b). Knockdown of ZRF1 abolished the increased enrichment of p113/ZRF1/BRD4 complex on promoters of these target genes induced by over-expression of ecircCUX1, while silencing of BRD4 did not affect the binding of p113 or ZRF1 to target gene promoters (Additional file 1: Fig. S7a-c). Ectopic expression of ecircCUX resulted in increase of promoter activity, transcript and protein levels of ALDH3A1, NDUFA1, and NDUFAF5 in SH-SY5Y and SK-N-SH cells, which were eliminated after knockdown of ZRF1 or BRD4, respectively (Additional file 1: Fig. S7a-c and Fig. 4c). Consistent with the roles of $A L D H 3 A 1$ in converting lipid peroxidation product (4-HNE) into fatty acids [31] (Fig. 4d), immunostaining assay indicated upregulation of ALDH3A1 and downregulation of 4-HNE in NB cells stably over-expressing ecircCUX, which was rescued by silencing of $Z R F 1$ or BRD4 (Additional file 1: Fig. S8a). Notably, silencing of ZRF1 or BRD4 abolished the increase of long-chain fatty acid production, OCR levels, mitochondrial membrane potential, complex I activity, $\mathrm{NAD}^{+} / \mathrm{NADH}$ ratio, and ATP production in SH-SY5Y cells stably over-expressing ecircCUX1 (Fig. 4e, $\mathrm{f}$ and Additional file 1: Fig. S8b). Moreover, ectopic expression of ZRF1 increased the expression of $A L D H 3 A 1, N D U F A 1$, and NDUFAF5, and led to increase in OCR levels, mitochondrial membrane potential, complex I activity, $\mathrm{NAD}^{+} / \mathrm{NADH}$ ratio, ATP production, growth, and invasion of $\mathrm{BE}(2)-\mathrm{C}$ cells, while knockdown of $A L D H 3 A 1$, NDUFA1, or NDUFAF5 abolished these effects (Additional file 1: Fig. S9a-f). Taken together, these results suggested that p113/ZRF1/BRD4 complex promoted lipid metabolic reprogramming and mitochondrial complex I activity in NB cells.

\section{p113 facilitates tumorigenesis and aggressiveness of NB cells via interacting with ZRF1}

We further explored the cooperative roles of p113 and ZRF1 in NB progression. Knockdown of ZRF1 by CRISPRi significantly prevented the increase of anchorage-independent growth and invasion of NB cells induced by stable ecircCUX1 over-expression (Fig. 5a, b). There were increased growth, volume, weight, Ki-67 proliferation index, and CD31-positive intratmoral microvessels of subcutaneous xenografts in athymic nude mice formed by injection of SH-SY5Y

\footnotetext{
(See figure on next page.)

Fig. 5 p113 facilitates tumorigenesis and aggressiveness of NB cells via interacting with ZRF1. a and b Representative images (left panel) and quantification (right panel) of soft agar (a) and matrigel invasion (b) assays indicating the growth and invasion of NB cells stably transfected with empty vector (mock) or ecircCUX1, and those co-transfected with sgRNA specific against ZRF1 for CRISPRi. c Representative images (upper panel), in vivo growth curve, and weight at the end points (lower panel) of xenografts in nude mice formed by subcutaneous injection of SH-SY5Y cells stably transfected with mock or ecircCUX1, and those co-transfected with sgRNA specific against ZRF1 for CRISPRi ( $n=5$ for each group). $d$ Representative images (upper panel) and quantification (lower panel) of immunohistochemical staining showing expression of Ki-67 and CD31 within xenografts ( $n=5$ for each group). Scale bars: $50 \mu \mathrm{m}$. e and $\mathbf{f}$ Representative in vivo images (e), hematoxylin \& eosin staining (f, upper panel), lung metastatic counts (f, lower panel), and Kaplan-Meier curves (f, lower panel) of nude mice treated with tail vein injection of SH-SY5Y cells stably transfected with mock or ecircCUX1, and those co-transfected with sgRNA specific against ZRF1 for CRISPRi ( $n=5$ for each group). Scale bars: $100 \mu \mathrm{m}$. ANOVA compared the difference in a-d and $\mathbf{f}$. Log-rank test for survival comparison in $\mathbf{f} .{ }^{*} P<0.05$ vs. mock+CRISPRi-Scb. Data are shown as mean \pm s.e.m. (error bars) and representative of three independent experiments in $\mathbf{a}$ and $\mathbf{b}$
} 
a

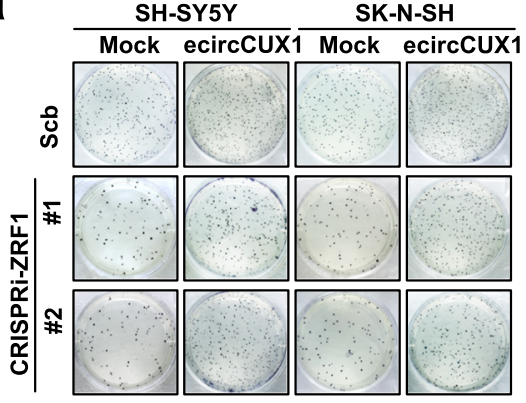

$\overline{\overline{0}} 6007$ Mock+CRISPRi-Scb

Mock+CRISPRi-ZRF1 \#1

ㅁ 2 ecircCUX1+CRISPRi-Scb

\& $400-\square$ ecircCUX1+CRISPRi-ZRF1 \#1

400-

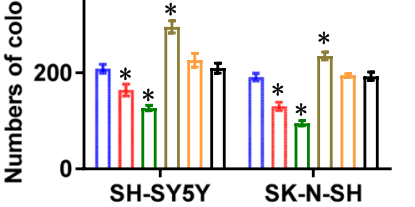

C
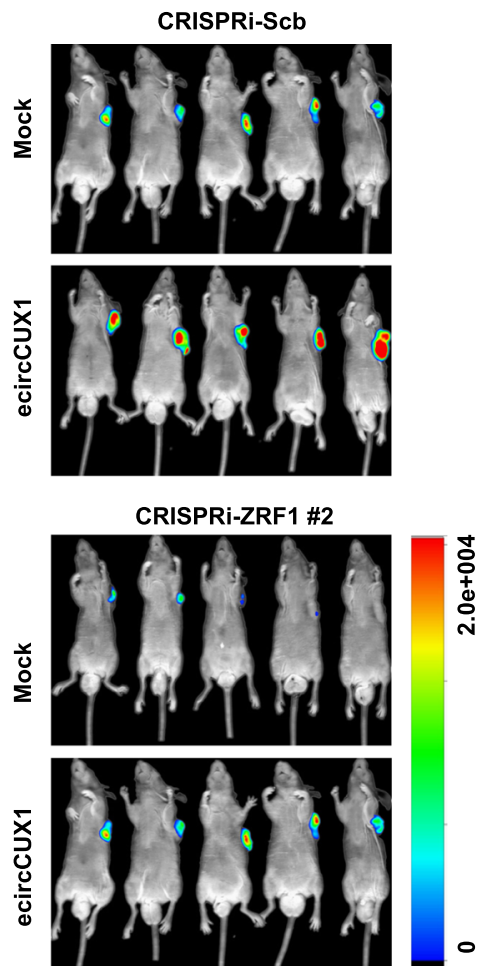

b SH-SY5Y SK-N-SH
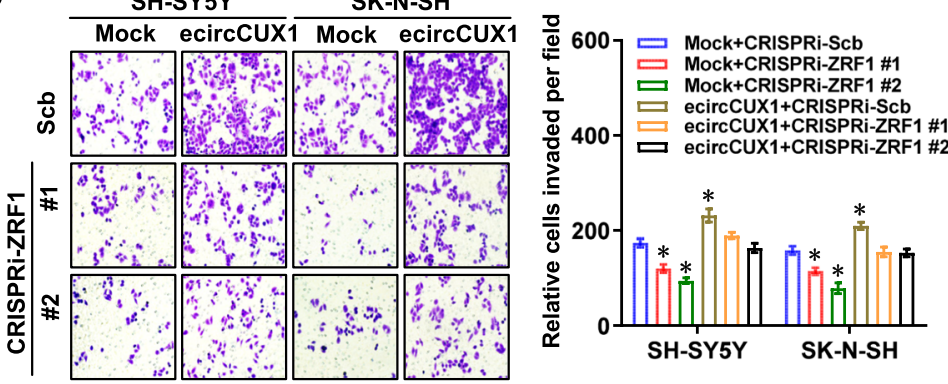

d $\frac{\text { CRISPRi-Scb }}{\text { Mock ecircCUX1 }} \frac{}{\text { Mock enISPRi-ZRF1 \#2 }}$
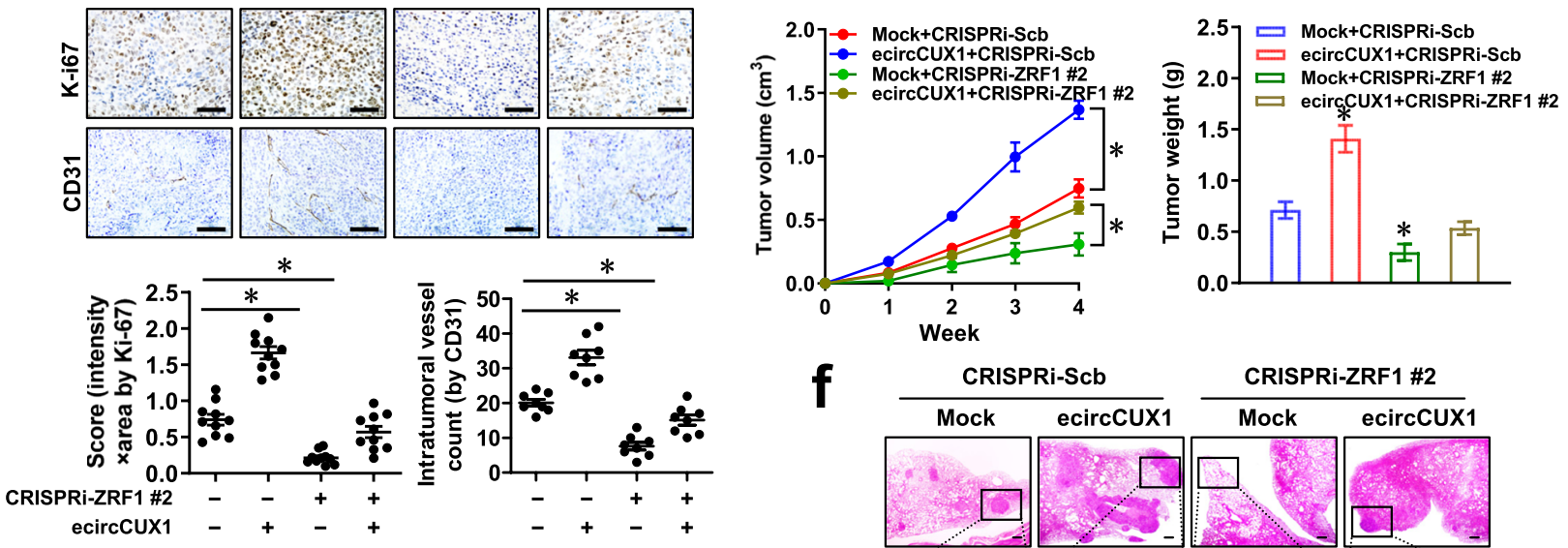

\section{e}
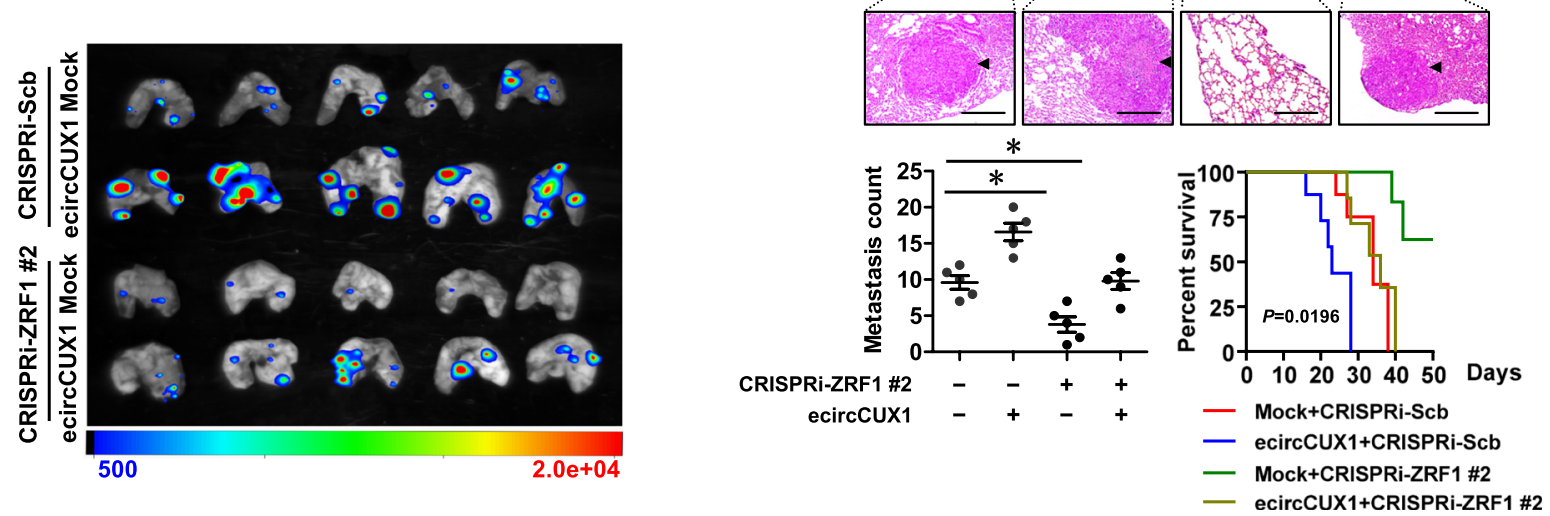

Fig. 5 (See legend on previous page.) 
cells stably transfected with ecircCUX1, and these effects were eliminated after knockdown of ZRF1 (Fig. 5c, d). Athymic nude mice treated with tail vein injection of SH-SY5Y cells stably transfected with ecircCUX1 presented more lung metastatic colonies and lower survival probability, which was abolished by silencing of ZRF1 (Fig. 5e, f). These results suggested that $\mathrm{p} 113$ facilitated the tumorigenesis and aggressiveness of NB cells via interacting with ZRF1.

\section{Therapeutic blocking p113-ZRF1 interaction inhibits NB progression}

Based on the importance of SANT domain of ZRF1 in interacting with p113, we designed cell-penetrating peptides using Peptiderive server [32], named as ZRF1 inhibitory peptide with 12 amino acids in length (ZIP-12) (Fig. 6a). Incubation of NB cells with ZIP-12, but not with mutant control peptide, led to obvious aggregation within nucleus (Fig. 6b). ZIP-12 was able to bind to endogenous p113 protein (Additional file 1: Fig. S10a) and abolish the interaction between p113 and ZRF1 in NB cells, without impact on that of p113 and BRD4 (Fig. 6c). In addition, treatment with ZIP-12, but not of mutant control peptide, inhibited the ZRF1 enrichment, promoter activity, and expression of $A L D H 3 A 1, N D U F A 1$, and NDUFAF5 in NB cells (Additional file 1: Fig. S10b-e). As a result, there was reduced long-chain fatty acid levels, mitochondrial membrane potential, complex I activity, $\mathrm{NAD}^{+} / \mathrm{NADH}$ ratio, ATP production in ZIP-12treated BE(2)-C cells (Additional file 1: Fig. S10f and Fig. 6d). Administration of ZIP-12 suppressed the viability of $B E(2)-C$ and IMR32 cells in a dose- and time-dependent manner, without impact on that of non-transformed normal MCF 10A cells (Additional file 1: Fig. S11a, b). Moreover, ZIP-12 repressed anchorage-independent growth and invasion of BE(2)-C and IMR32 cells (Additional file 1: Fig. S11c). Intravenous administration of ZIP-12 suppressed the fluorescence signals, volume, weight, Ki-67 proliferation index, and CD31-positive microvessels of subcutaneous xenograft tumors formed by $\mathrm{BE}(2)-\mathrm{C}$ cells in nude mice (Fig. 6e and Additional file 1: Fig. S11d). Moreover, intravenous administration of ZIP-12 led to less lung metastatic colonies and prolonged the survival time of athymic nude mice treated with tail vein injection of $\mathrm{BE}(2)-\mathrm{C}$ cells (Fig. $6 \mathrm{f}$ and Additional file 1: Fig. S11e). Collectively, these results demonstrated that blocking p113-ZRF1 interaction suppressed NB progression.

\section{CUX1, ZRF1, BRD4 and target genes are associated with poor outcome of tumor patients}

In 498 well-defined NB cases (GSE62564), KaplanMeier survival plots revealed that higher expression of p113 host gene CUX1 $\left(P=6.6 \times 10^{-3}\right), Z R F 1$ $\left(P=3.4 \times 10^{-16}\right), \quad$ BRD4 $\quad\left(P=9.3 \times 10^{-4}\right), \quad A L D H 3 A 1$ $\left(P=5.7 \times 10^{-5}\right), \quad N D U F A 1 \quad\left(P=2.5 \times 10^{-14}\right)$, or NDUFAF5 $\left(P=3.0 \times 10^{-3}\right)$ was associated with lower overall survival probability of patients (Fig. 7a). High expression of $Z R F 1$ or $B R D 4$ was also associated with poor survival of patients with breast cancer, colon cancer, Ewing sarcoma, glioma, melanoma, myeloma, or renal cancer (Additional file 1: Fig. S12). There was a positive expression correlation between $Z R F 1$ and $A L D H 3 A 1(R=0.131$, $\left.P=3.4 \times 10^{-3}\right)$, NDUFA1 $\left(R=0.334, P=2.0 \times 10^{-14}\right)$, or NDUFAF5 $\left(R=0.315, P=6.6 \times 10^{-13}\right)$ in $498 \mathrm{NB}$ specimens (Fig. $7 \mathrm{~b}$ ). These results indicated that expression of $C U X 1, Z R F 1, B R D 4$, or target genes was associated with poor outcome of tumors.

\section{Discussion}

As a homeodomain transcription factor, CUX1 regulates cell cycle progression, proliferation, migration, and invasiveness of numerous tumor cell lines [33]. The major isoforms of CUX1 include full-length p200, p110 proteolytically processed from p200, and CASP [34]. Notably, p200 binds rapidly but only transiently to

\footnotetext{
(See figure on next page.)

Fig. 6 Therapeutic blocking p113-ZRF1 interaction inhibits NB progression. a 3D structure and sequences of inhibitory peptides (ZIP-12) for blocking interaction between $\mathrm{p} 113$ and ZRF1, and those of mutant control (Ctrl) peptides. b Confocal images showing the distribution of synthesized FITC-labeled Ctrl or ZIP-12 peptides $\left(20 \mathrm{\mu mol} \cdot \mathrm{L}^{-1}\right.$, arrowheads) within cultured BE(2)-C cells, with nuclei and cellular membranes staining with DAPI or Dil. Scale bars: $10 \mu \mathrm{m}$. c Co-IP and western blot assays indicating the interaction of p113 with ZRF1 or BRD4 in BE(2)-C cells treated with Ctrl or ZIP-12 peptides $\left(20 \mu \mathrm{mol} \cdot \mathrm{L}^{-1}\right)$ for $24 \mathrm{~h}$. d Relative fatty acid levels, complex I activity, NAD $/ \mathrm{NADH}$ ratio, and ATP levels in BE(2)-C cells treated with Ctrl or ZIP-12 peptides $\left(20 \mu \mathrm{mol} \cdot \mathrm{L}^{-1}\right)$ for $24 \mathrm{~h}$. e In vivo images (left upper panel), growth curve (right panel), and weight at the end points (right panel) of xenografts formed by subcutaneous injection of BE(2)-C cells in nude mice ( $n=5$ per group) that were treated with intravenous injection of Ctrl or ZIP-12 peptides $\left(5 \mathrm{mg} \cdot \mathrm{kg}^{-1}\right.$ ) as indicated (left lower panel). $\mathbf{f}$ In vivo imaging (left panel), lung metastatic colonization (right lower panel), and Kaplan-Meier curves (right lower panel) of nude mice ( $n=5$ for each group) treated with tail vein injection of BE(2)-C cells, Ctrl or ZIP-12 peptides ( $5 \mathrm{mg} \cdot \mathrm{kg}^{-1}$ ) as indicated (right upper panel). Student's $t$ test or ANOVA compared the difference in d-f. Log-rank test for survival comparison in $\mathbf{f} .{ }^{*} P<0.05,{ }^{* *} P<0.01$ vs. Ctrl. Data are shown as mean \pm s.e.m. (error bars) and representative of three independent experiments in $\mathbf{b}-\mathbf{d}$
} 
a

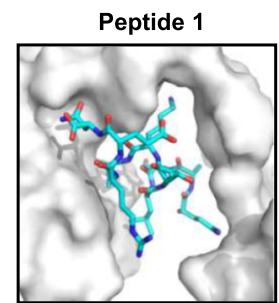

Ctrl \#1: Biotin-YGRKKRRQRRRPLRKDEYKLLEQK-FITC ZIP-12 \#1: Biotin-YGRKKRRQRRRPWTTEEQKLLEQK-FITC Ctrl \#2: Biotin-YGRKKRRQRRRLKTYPQSYRLCWK-FITC ZIP-12 \#2: Biotin-YGRKKRRQRRRLKTYPVNTPERWK-FITC

C

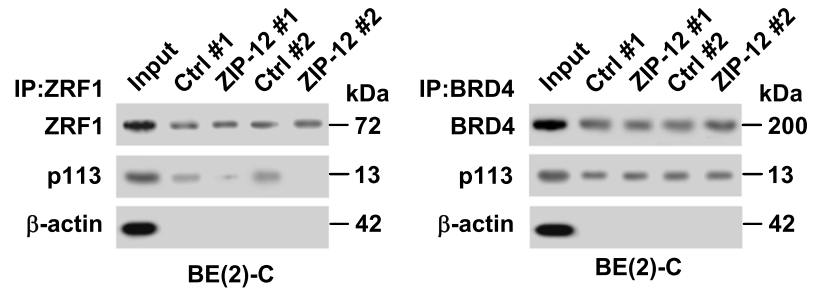

b
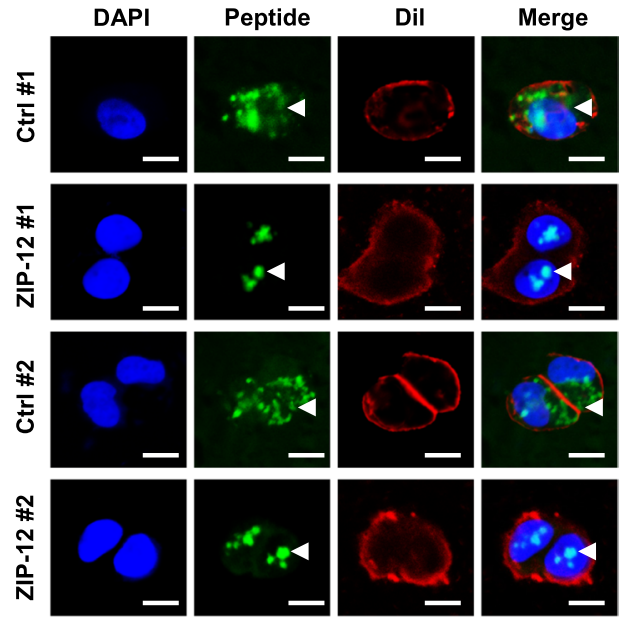

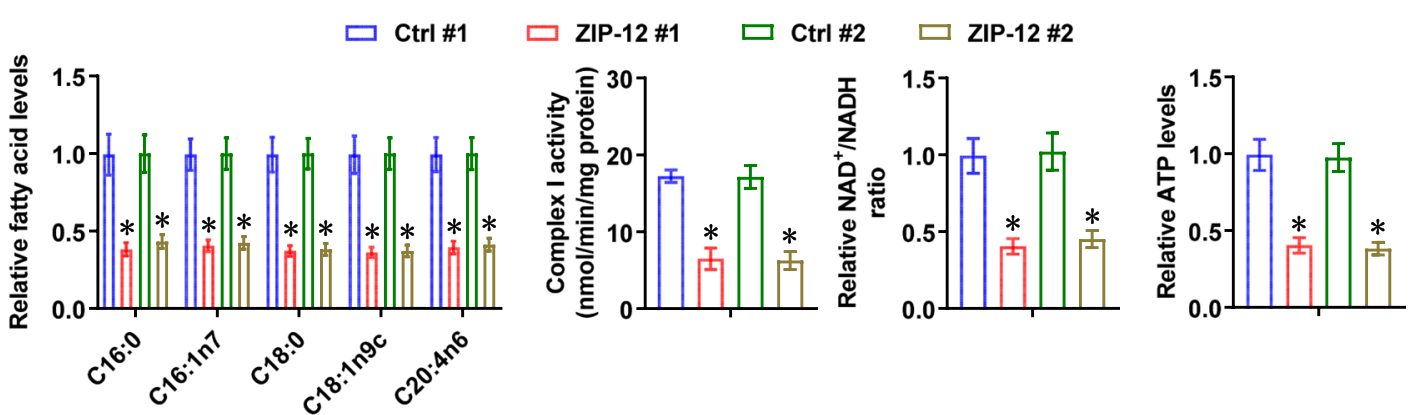

e
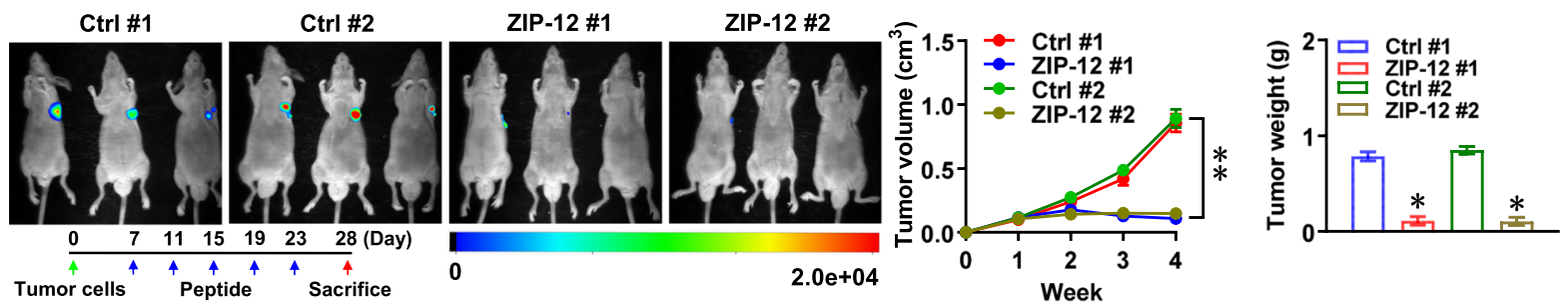

f

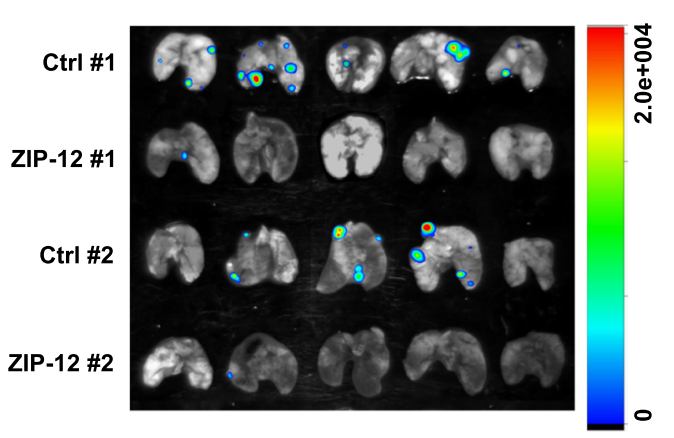

Fig. 6 (See legend on previous page.)

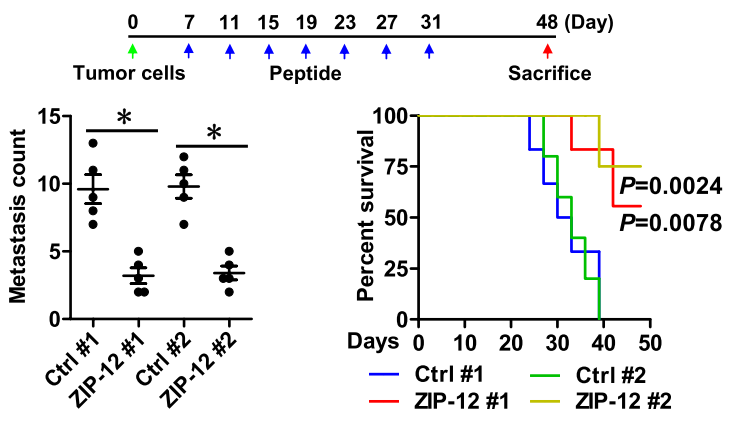


DNA, while p110 isoform stably interacts with DNA to regulate gene transcription [35]. Meanwhile, CASP is a Golgi membrane protein related to giantin [36]. In our previous study, we have demonstrated oncogenic roles of CUX1 and its generated circRNA in aerobic glycolysis and tumor progression [19]. In this study, through highthroughput quantitative proteomics, we discovered that a new CUX1 isoform (p113), but not p200, p110 or CASP, was induced by serum loss stress in NB cells. As a protein encoded by ecircCUX1, p113 was upregulated in NB tissues and cell lines, and associated with poor survival of NB patients. Gain- and loss-of-function studies indicated that $\mathrm{p} 113$ promoted the lipid metabolism, mitochondrial complex I activity, growth, and invasiveness of NB cells (Fig. 7c), suggesting its oncogenic roles in NB progression.

In tumor cells, there was spontaneous fatty acid peroxidation mediated by reactive oxygen species, resulting in production of fatty aldehydes [37]. As an unique process, ALDH3A1 catalyzes conversion of fatty aldehydes to fatty acids with NADH production as an extra source of electrons, resulting in mitochondrial FAO and ATP synthesis [38]. It is well known that mitochondrial ETC is necessary for tumor growth [39]. As the largest component of mitochondrial respiratory chain, complex I is the first entry point of electrons into oxidative phosphorylation, and donates electrons to ubiquinone, resulting in generation of ubiquinol and $\mathrm{NAD}^{+}$to maintain cellular $\mathrm{NAD}^{+} / \mathrm{NADH}$ balance [40]. As a component of complex I, NDUFA1 is essential for respiratory activity [41] and tumor growth [42]. NDUFAF5 is involved in building up complex I and electrochemical potential required for ATP production [43]. In this study, we demonstrated that p113 induced transcriptional upregulation of ALDH3A1, NDUFA1, and NDUFAF5, leading to increased conversion of fatty aldehydes into fatty acids, fatty acid $\beta$-oxidation, and mitochondrial complex I activity for ATP production, providing a novel mechanism regulating lipid metabolic reprogramming and mitochondrial activity in NB. In addition, our evidence indicated that p113 directly interacted with transcription factor ZRF1. Since knockdown of ZRF1 abolished the p113-facilitated aggressive behavior of NB cells, our findings indicate that tumor promoting functions of p113 are mediated, at least in part, through interacting with ZRF1.

ZRF1 is a transcription factor recognizing target genes via DNAJ or SANT domain [44], and is involved in various biological processes such as embryonic development, cell differentiation, neural progenitor cell maintenance, apoptosis, and cellular proliferation [45]. In addition, ZRF1 attenuates the recruitment of polycomb repressive complex 1 (PRC1) on gene promoters by binding to mono-ubiquitinated histone $\mathrm{H} 2 \mathrm{~A}$, resulting in de-repression of PRC1 downstream genes [46]. Elevated ZRF1 expression is documented in many tumors, and is associated with poor survival of tumor patients [45, 47], suggesting its oncogenic roles in tumor progression. As a transcriptional co-activator, BRD4 is one member of BD domain and ET domain family, and preferentially interacts with acetylated lysine of histones to recruit distinct transcriptional regulators, leading to initiation and elongation of gene transcription [48]. BRD4 plays a pivotal role in embryogenesis and cancer development [49], while its inhibitor is able to eliminate transcriptional activation of oncogenes and suppress tumor progression [50]. In this study, we found that p113/ZRF1/BRD4 transcriptional trimer was essential for upregulation of genes involved in lipid metabolic reprogramming and mitochondrial activity. Meanwhile, silencing of ZRF1 abolished the enrichment of this complex on target gene promoters. Importantly, an inhibitory peptide blocking p113-ZRF1 interaction was efficient in suppressing lipid metabolic reprogramming, mitochondrial activity, tumorigenesis, and aggressiveness of NB cells.

\section{Conclusions}

In summary, we demonstrate that as a protein encoded by circRNA derived from CUX1, p113 is highly expressed in NB tissues and cells, and facilitates growth, invasion, and metastasis of NB cells. Mechanistically, p113 interacts with ZRF1 and BRD4 to form

\footnotetext{
(See figure on next page.)

Fig. 7 CUX1, ZRF1, BRD4 and target genes are associated with poor outcome of NB patients. a Kaplan-Meier curves indicating overall survival of 498 well-defined NB cases (GSE62564) with high or low expression of CUX1 (cutoff value =32.233), ZRF1 (cutoff value =21.749), BRD4 (cutoff value $=652.58$ ), ALDH3A1 (cutoff value =1.181), NDUFA1 (cutoff value $=22.511$ ), or NDUFAF5 (cutoff value $=7.964$ ). $\mathbf{b}$ The positive expression correlation between ZRF1 and ALDH3A1, NDUFA1, or NDUFAF5 in 498 well-defined NB cases (GSE62564). c The mechanisms underlying p113-faciliated NB progression: as a novel protein encoded by ecircCUX1, p113 cooperates with ZRF1 and BRD4 to activate the transcription of ALDH3A1, NDUFA1, or NDUFAF5, resulting in promoted conversion of fatty aldehydes into fatty acids, fatty acid $\beta$-oxidation, mitochondrial complex I activity, growth, and aggressiveness of NB cells. Meanwhile, inhibitory peptides (ZIP-12) blocking p113-ZRF1 interaction suppresses tumor progression. Log-rank test for survival comparison in $\mathbf{a}$. Pearson's correlation coefficient for $\mathbf{b}$
} 

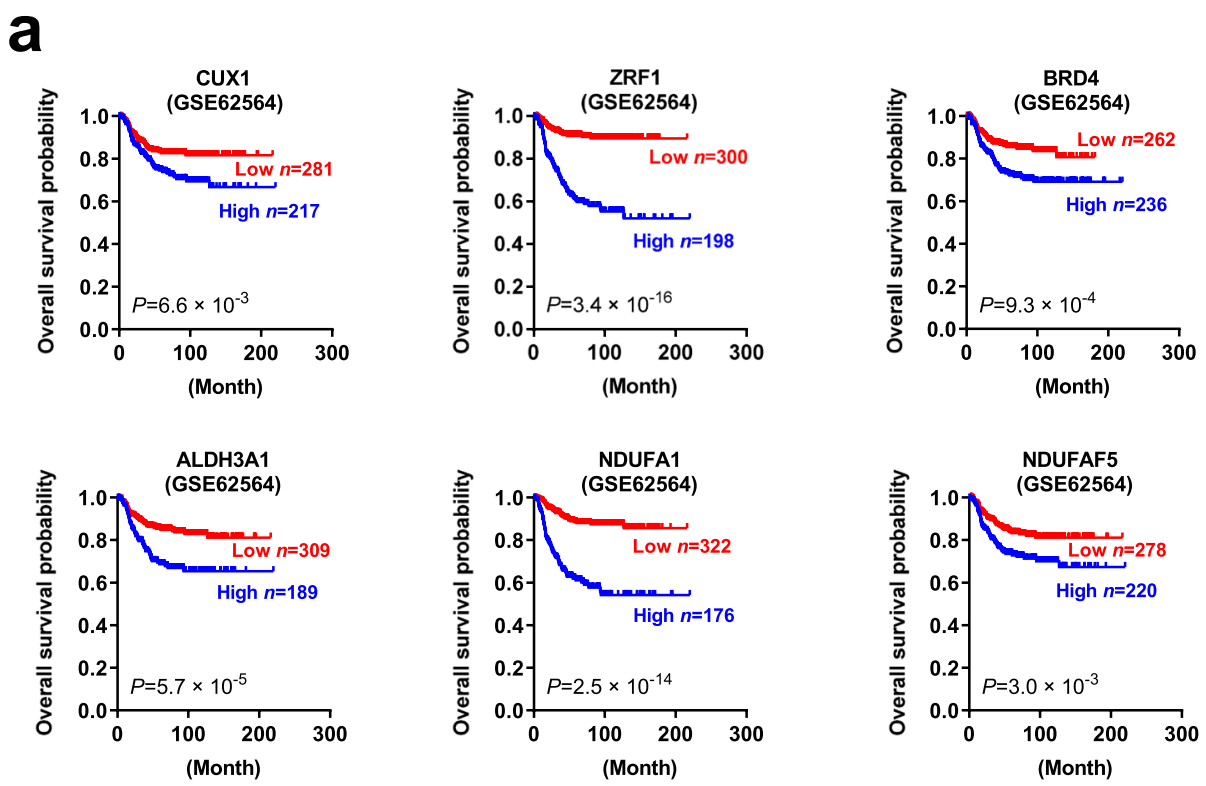

b
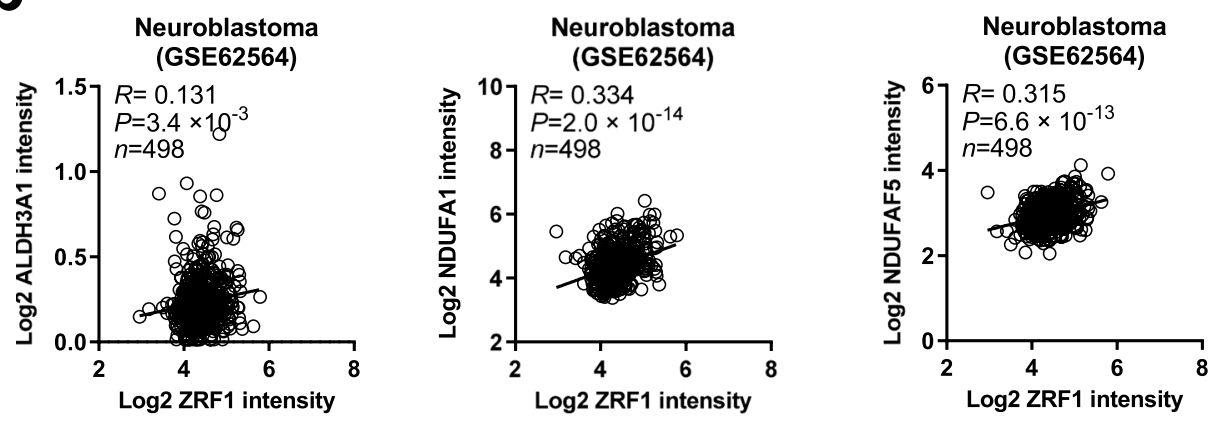

C

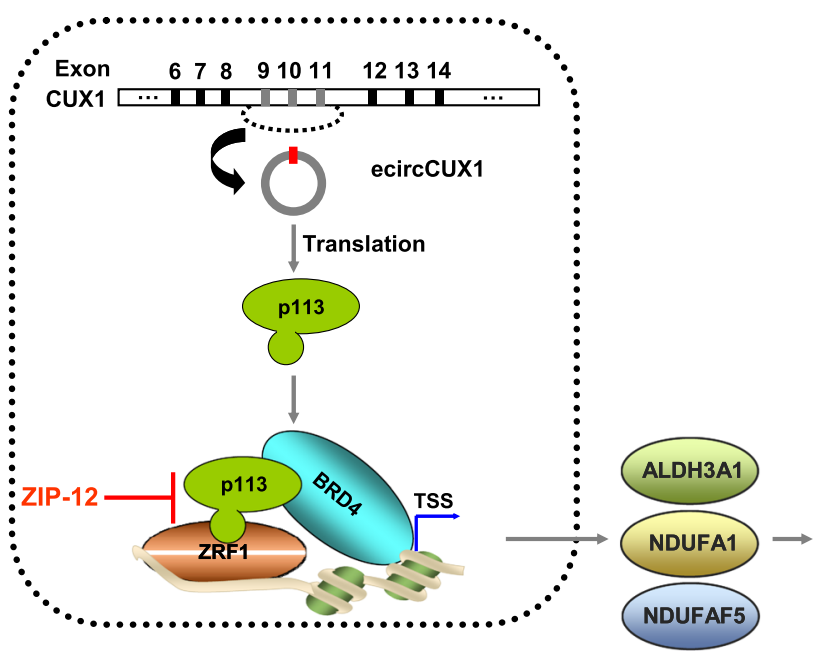

Lipids

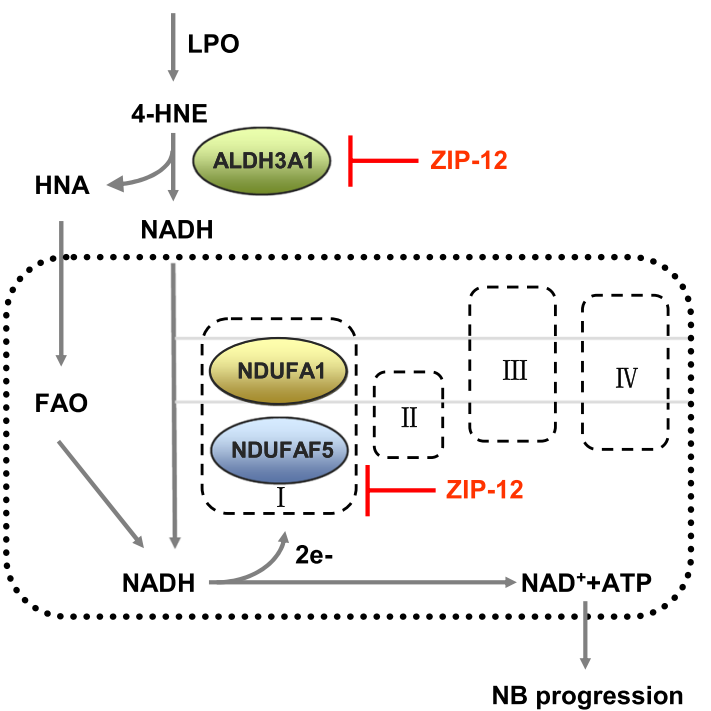

Fig. 7 (See legend on previous page.) 
a transcriptional regulatory complex, resulting in transcriptional activation of $A L D H 3 A 1, N D U F A 1$, and NDUFAF5, thus enhancing lipid metabolic reprogramming and mitochondrial activity of NB cells. Importantly, in clinical NB cases, high p113 expression is associated with unfavorable survival probability. The inhibitory peptides blocking p113-ZRF1 interaction suppresses the tumorigenesis and aggressiveness of NB cells. We believe that this study extends our knowledge about the regulation of lipid metabolic reprogramming and mitochondrial activity by circRNA-coding protein, and reveals p113/ZRF1/BRD4 axis as a potential therapeutic target for NB progression.

\begin{abstract}
Abbreviations
4-HNE: 4-Hydroxynonenal; ALDH3A1: Aldehyde dehydrogenase 3 family member A1; BiFC: Bimolecular fluorescence complementation; BRD4: Bromodomain protein 4; circRNA: Circular RNA; co-IP: Co-immunoprecipitation; CRISPRi: CRISPR interference; CUX1: CUT-like homeobox 1; ETC: Electron transport chain; FAO: Fatty acid $\beta$-oxidation; IRES: Internal ribosome entry site; $\mathrm{NAD}^{+}$: Nicotinamide adenine dinucleotide; $\mathrm{NADH}$ : Nicotinamide adenine dinucleotide; NB: Neuroblastoma; NDUFA1: NADH:ubiquinone oxidoreductase subunit A1; NDUFAF5: NADH:ubiquinone oxidoreductase complex assembly factor 5; OCR: Oxygen consumption rate; TCF3: Transcription factor 3; ZRF1: Zuotin-related factor $1 .$.
\end{abstract}

\section{Supplementary Information}

The online version contains supplementary material available at https://doi. org/10.1186/s12943-021-01421-8.

\section{Additional file 1: Fig. S1. Coding ability of ecircCUX1 in NB. Fig. S2.} Expression profiles and roles of ecircCUX1-encoded p113. Fig. S3. Effects of ecircCUX1 or p113 on mitochondrial mass and structure in NB cells. Fig. S4. ecircCUX1 promotes fatty acid oxidation and growth of NB via encoding p113. Fig. S5. ecircCUX1 facilitates mitochondrial complex I activity and aggressiveness of NB in vivo. Fig. S6. Interaction of p113 with ZRF1 and BRD4 in NB cells. Fig. S7. ecircCUX1 facilitates expression of ALDH3A1, NDUFA1, or NDUFAF5 in NB cells. Fig. S8. ecircCUX1 reduces peroxidated lipids and increases mitochondrial membrane potential via ZRF1 or BRD4 in NB cells. Fig. S9. ZRF1 promotes mitochondrial complex I activity, growth, and aggressiveness of NB cells via target genes. Fig. S10. Effects of inhibitory peptides blocking p113-ZRF1 interaction. Fig. S11. ZIP-12 inhibits viabilities, growth, invasion, and metastasis of NB cells. Fig. S12. Kaplan-Meier curves of ZRF1 and BRD4 in multiple cancers. Table S1. Primer sets used for RT-PCR, APCR and ChIP. Table S2. Oligonucleotide sets used for constructs. Table S3. Oligonucleotide sets used for short hairpin RNAs and CRISPR-Cas9/dCas9. Table S4. Mass spectrometry analysis of proteins altered by serum deprivation. Table S5. p113 expression in human NB tissues. Table S6. Mass spectrometry analysis of proteins pulled down by p113 antibody. Table S7. Mass spectrometry analysis of proteins pulled down by Flag antibody.

\section{Acknowledgements}

We are grateful for Dr. Guosong Jiang for providing vectors.

\section{Authors' contributions}

F.Y. and A.H. conceived and performed most of the experiments; Y.G., J.W., D.L., X.W., and S.J. accomplished some of in vitro experiments; B.Y., S.C., Y.Z., Q.L., G.C., and H.G. accomplished in vivo studies; F.Y. and A.H. undertook the mining of publicly available datasets; F.Y., Q.T., and L.Z. wrote the manuscript. All authors read and approved the final manuscript.

\section{Funding}

This work was granted by the National Natural Science Foundation of China (81773094, 81772967, 81874085, 81874066, 81802925, 81903011, 81903008, 82072801, 82173316).

\section{Availability of data and materials}

The data supporting the conclusions of this article are presented within article and its additional files.

\section{Declarations}

\section{Ethics approval and consent to participate}

All animal experiments were carried out in accordance with NIH Guidelines for the Care and Use of Laboratory Animals, and approved by the Animal Care Committee of Tongji Medical College (approval number:Y20080290). Human tissue study (approval number: 2011-S085) was approved by Institutional Review Board of Tongji Medical College.

\section{Consent for publication}

Written informed consent was obtained from all patients.

\section{Competing interests}

The authors declare that they have no competing interests.

\section{Author details}

${ }^{1}$ Department of Pediatric Surgery, Union Hospital, Tongji Medical College, Huazhong University of Science and Technology, 1277 Jiefang Avenue, Wuhan, Hubei Province 430022, People's Republic of China. ${ }^{2}$ Clinical Center of Human Genomic Research, Union Hospital, Tongji Medical College, Huazhong University of Science and Technology, 1277 Jiefang Avenue, Wuhan, Hubei Province 430022, People's Republic of China. ${ }^{3}$ Department of Geriatrics, Union Hospital, Tongji Medical College, Huazhong University of Science and Technology, 1277 Jiefang Avenue, Wuhan, Hubei Province 430022, People's Republic of China. ${ }^{4}$ Department of Pathology, Union Hospital, Tongji Medical College, Huazhong University of Science and Technology, 277 Jiefang Avenue, Wuhan, Hubei Province 430022, People's Republic of China. ${ }^{5}$ Department of Gastrointestinal Surgery, Union Hospital, Tongji Medical College, Huazhong University of Science and Technology, 1277 Jiefang Avenue, Wuhan, Hubei Province 430022, People's Republic of China.

Received: 21 June 2021 Accepted: 8 September 2021

Published online: 27 September 2021

\section{References}

1. Pinto NR, Applebaum MA, Volchenboum SL, Matthay KK, London WB, Ambros PF, et al. Advances in risk classification and treatment strategies for neuroblastoma. J Clin Oncol. 2015;33:3008-17.

2. Budhu A, Roessler S, Zhao X, Yu Z, Forgues M, Ji J, et al. Integrated metabolite and gene expression profiles identify lipid biomarkers associated with progression of hepatocellular carcinoma and patient outcomes. Gastroenterology. 2013;144:1066-75.

3. Zaugg K, Yao Y, Reilly PT, Kannan K, Kiarash R, Mason J, et al. Carnitine palmitoyltransferase $1 \mathrm{C}$ promotes cell survival and tumor growth under conditions of metabolic stress. Genes Dev. 2011;25:1041-51.

4. Ma Y, Temkin SM, Hawkridge AM, Guo C, Wang W, Wang XY, et al. Fatty acid oxidation: an emerging facet of metabolic transformation in cancer. Cancer Lett. 2018;435:92-100.

5. Caro P, Kishan AU, Norberg E, Stanley IA, Chapuy B, Ficarro SB, et al. Metabolic signatures uncover distinct targets in molecular subsets of diffuse large B cell lymphoma. Cancer Cell. 2012;22:547-60.

6. Lasda E, Parker R. Circular RNAs: diversity of form and function. RNA 2014;20:1829-42

7. Memczak S, Jens M, Elefsinioti A, Torti F, Krueger J, Rybak A, et al. Circular RNAs are a large class of animal RNAs with regulatory potency. Nature. 2013;495:333-8.

8. Li Z, Huang C, Bao C, Chen L, Lin M, Wang X, et al. Exon-intron circular RNAs regulate transcription in the nucleus. Nat Struct Mol Biol. 2015:22:256-64. 
9. Yang $Q$, Du WW, Wu N, Yang W, Awan FM, Fang L, et al. A circular RNA promotes tumorigenesis by inducing c-myc nuclear translocation. Cell Death Differ. 2017;24:1609-20.

10. Yang Y, Gao X, Zhang M, Yan S, Sun C, Xiao F, et al. Novel role of FBXW7 circular RNA in repressing glioma tumorigenesis. J Natl Cancer Inst. 2018;110:304-15.

11. Liang WC, Wong CW, Liang PP, Shi M, Cao Y, Rao ST, et al. Translation of the circular RNA circ $\beta$-catenin promotes liver cancer cell growth through activation of the Wnt pathway. Genome Biol. 2019:20:84.

12. Xia X, Li X, Li F, Wu X, Zhang M, Zhou H, et al. A novel tumor suppressor protein encoded by circular AKT3 RNA inhibits glioblastoma tumorigenicity by competing with active phosphoinositide-dependent Kinase-1. Mol Cancer. 2019;18:131.

13. Zheng $X$, Chen $L$, Zhou Y, Wang $Q$, Zheng Z, Xu B, et al. A novel protein encoded by a circular RNA circPPP1R12A promotes tumor pathogenesis and metastasis of colon cancer via hippo-YAP signaling. Mol Cancer. 2019;18:47

14. Zhao X, Li D, Pu J, Mei H, Yang D, Xiang X, et al. CTCF cooperates with noncoding RNA MYCNOS to promote neuroblastoma progression through facilitating MYCN expression. Oncogene. 2016;35:3565-76.

15. Li D, Song H, Mei H, Fang E, Wang X, Yang F, et al. Armadillo repeat containing 12 promotes neuroblastoma progression through interaction with retinoblastoma binding protein 4. Nat Commun. 2018;9:2829.

16. Fang $E$, Wang $X$, Yang F, Hu A, Wang J, Li D, et al. Therapeutic targeting of MZF1-AS1/PARP1/E2F1 axis inhibits proline synthesis and neuroblastoma progression. Adv Sci. 2019;6:1900581.

17. Wu X, Liu D, Tao D, Xiang W, Xiao X, Wang M, et al. BRD4 regulates EZH2 transcription through upregulation of C-MYC and represents a novel therapeutic target in bladder cancer. Mol Cancer Ther. 2016;15:1029-42.

18. Yang F, Hu A, Li D, Wang J, Guo Y, Liu Y, et al. Circ-HuR suppresses HuR expression and gastric cancer progression by inhibiting CNBP transactivation. Mol Cancer. 2019;18:158

19. Li H, Yang F, Hu A, Wang X, Fang E, Chen Y, et al. Therapeutic targeting of circ-CUX1/EWSR1/MAZ axis inhibits glycolysis and neuroblastoma progression. EMBO Mol Med. 2019;11:e10835.

20. Nottrott S, Simard MJ, Richter JD. Human let-7a miRNA blocks protein production on actively translating polyribosomes. Nat Struct Mol Biol. 2006;13:1108-14.

21. Agnello M, Morici G, Rinaldi AM. A method for measuring mitochondrial mass and activity. Cytotechnology. 2008;56:145-9.

22. Long NP, Min JE, Anh NH, Kim SJ, Park S, Kim HM, et al. Isolation and metabolic assessment of cancer cell mitochondria. ACS Omega. 2020;5:27304-13.

23. Li D, Mei H, Pu J, Xiang X, Zhao X, Qu H, et al. Intelectin 1 suppresses the growth, invasion and metastasis of neuroblastoma cells through up-regulation of $\mathrm{N}$-myc downstream regulated gene 2. Mol Cancer. 2015;14:47.

24. Song H, Li D, Wang X, Fang E, Yang F, Hu A, et al. HNF4A-AS1/hnRNPU/ CTCF axis as a therapeutic target for aerobic glycolysis and neuroblastoma progression. J Hematol Oncol. 2020;13:24.

25. Huang D, Li T, Wang L, Zhang L, Yan R, Li K, et al. Hepatocellular carcinoma redirects to ketolysis for progression under nutrition deprivation stress. Cell Res. 2016;26:1112-30.

26. Munir R, Lisec J, Swinnen JV, Zaidi N. Lipid metabolism in cancer cells under metabolic stress. Br J Cancer. 2019;120:1090-8.

27. Lachmann A, Xu H, Krishnan J, Berger SI, Mazloom AR, Ma'ayan A. ChEA: transcription factor regulation inferred from integrating genome-wide ChIP-X experiments. Bioinformatics. 2010;26:2438-44.

28. Medvedeva YA, Lennartsson A, Ehsani R, Kulakovskiy IV, Vorontsov IE, Panahandeh P, et al. EpiFactors: a comprehensive database of human epigenetic factors and complexes. Database. 2015;2015:bav067.

29. Hu CD, Kerppola TK. Simultaneous visualization of multiple protein interactions in living cells using multicolor fluorescence complementation analysis. Nat Biotechnol. 2003;21:539-45.

30. Gilbert LA, Horlbeck MA, Adamson B, Villalta JE, Chen Y, Whitehead EH et al. Genome-scale CRISPR-mediated control of gene repression and activation. Cell. 2014;159:647-61.
31. Laurora S, Tamagno E, Briatore F, Bardini P, Pizzimenti S, Toaldo C, et al. 4-Hydroxynonenal modulation of p53 family gene expression in the SKN-BE neuroblastoma cell line. Free Radic Biol Med. 2005:38:215-25.

32. Sedan Y, Marcu O, Lyskov S, Schueler-Furman O. Peptiderive server: derive peptide inhibitors from protein-protein interactions. Nucleic Acids Res. 2016;44:W536-41.

33. Michl P, Ramjaun AR, Pardo OE, Warne PH, Wagner M, Poulsom R, et al. CUTL1 is a target of TGF (beta) signaling that enhances cancer cell motility and invasiveness. Cancer Cell. 2005;7:521-32.

34. Ramdzan ZM, Nepveu A. CUX1, a haploinsufficient tumour suppressor gene overexpressed in advanced cancers. Nat Rev Cancer. 2014;14:673-82.

35. Harada R, Vadnais C, Sansregret L, Leduy L, Bérubé G, Robert F, et al. Genome-wide location analysis and expression studies reveal a role for p110 CUX1 in the activation of DNA replication genes. Nucleic Acids Res. 2008:36:189-202.

36. Gillingham AK, Pfeifer AC, Munro S. CASP, the alternatively spliced product of the gene encoding the CCAAT-displacement protein transcription factor, is a Golgi membrane protein related to giantin. Mol Biol Cell. 2002:13:3761-74.

37. Yang WS, SriRamaratnam R, Welsch ME, Shimada K, Skouta R, Viswanathan VS, et al. Regulation of ferroptotic cancer cell death by GPX4. Cell. 2014;156:317-31.

38. Lee JS, Kim SH, Lee S, Kang JH, Lee SH, Cheong JH, et al. Gastric cancer depends on aldehyde dehydrogenase $3 \mathrm{~A} 1$ for fatty acid oxidation. Sci Rep. 2019;9:16313.

39. Viale A, Pettazzoni P, Lyssiotis CA, Ying H, Sánchez N, Marchesini M, et al. Oncogene ablation-resistant pancreatic cancer cells depend on mitochondrial function. Nature. 2014;514:628-32.

40. Sharma LK, Lu J, Bai Y. Mitochondrial respiratory complex I: structure, function and implication in human diseases. Curr Med Chem. 2009;16:1266-77.

41. Wallace DC. Mitochondrial diseases in man and mouse. Science. 1999;283:1482-8

42. Martínez-Reyes I, Cardona LR, Kong H, Vasan K, McElroy GS, Werner M, et al. Mitochondrial ubiquinol oxidation is necessary for tumour growth. Nature. 2020;585:288-92.

43. Sugiana C, Pagliarini DJ, McKenzie M, Kirby DM, Salemi R, Abu-Amero KK, et al. Mutation of C20orf7 disrupts complex I assembly and causes lethal neonatal mitochondrial disease. Am J Hum Genet. 2008;83:468-78.

44. Boyer LA, Latek RR, Peterson CL. The SANT domain: a unique histone-tailbinding module? Nat Rev Mol Cell Biol. 2004:5:158-63.

45. Demajo S, Uribesalgo I, Gutiérrez A, Ballaré C, Capdevila S, Roth M, et al. ZRF1 controls the retinoic acid pathway and regulates leukemogenic potential in acute myeloid leukemia. Oncogene. 2014;33:5501-10.

46. Richly H, Rocha-Viegas L, Ribeiro JD, Demajo S, Gundem G, Lopez-Bigas $\mathrm{N}$, et al. Transcriptional activation of polycomb-repressed genes by ZRF1. Nature. 2010;468:1124-8.

47. Aloia L, Demajo S, Di Croce L. ZRF1: a novel epigenetic regulator of stem cell identity and cancer. Cell Cycle. 2015;14:510-5.

48. Filippakopoulos P, Picaud S, Mangos M, Keates T, Lambert JP, BarsyteLovejoy D, et al. Histone recognition and large-scale structural analysis of the human bromodomain family. Cell. 2012;149:214-31.

49. Donati B, Lorenzini E, Ciarrocchi A. BRD4 and Cancer: going beyond transcriptional regulation. Mol Cancer. 2018;17:164.

50. Lovén J, Hoke HA, Lin CY, Lau A, Orlando DA, Vakoc CR, et al. Selective inhibition of tumor oncogenes by disruption of super-enhancers. Cell. 2013:153:320-34

\section{Publisher's Note}

Springer Nature remains neutral with regard to jurisdictional claims in published maps and institutional affiliations. 